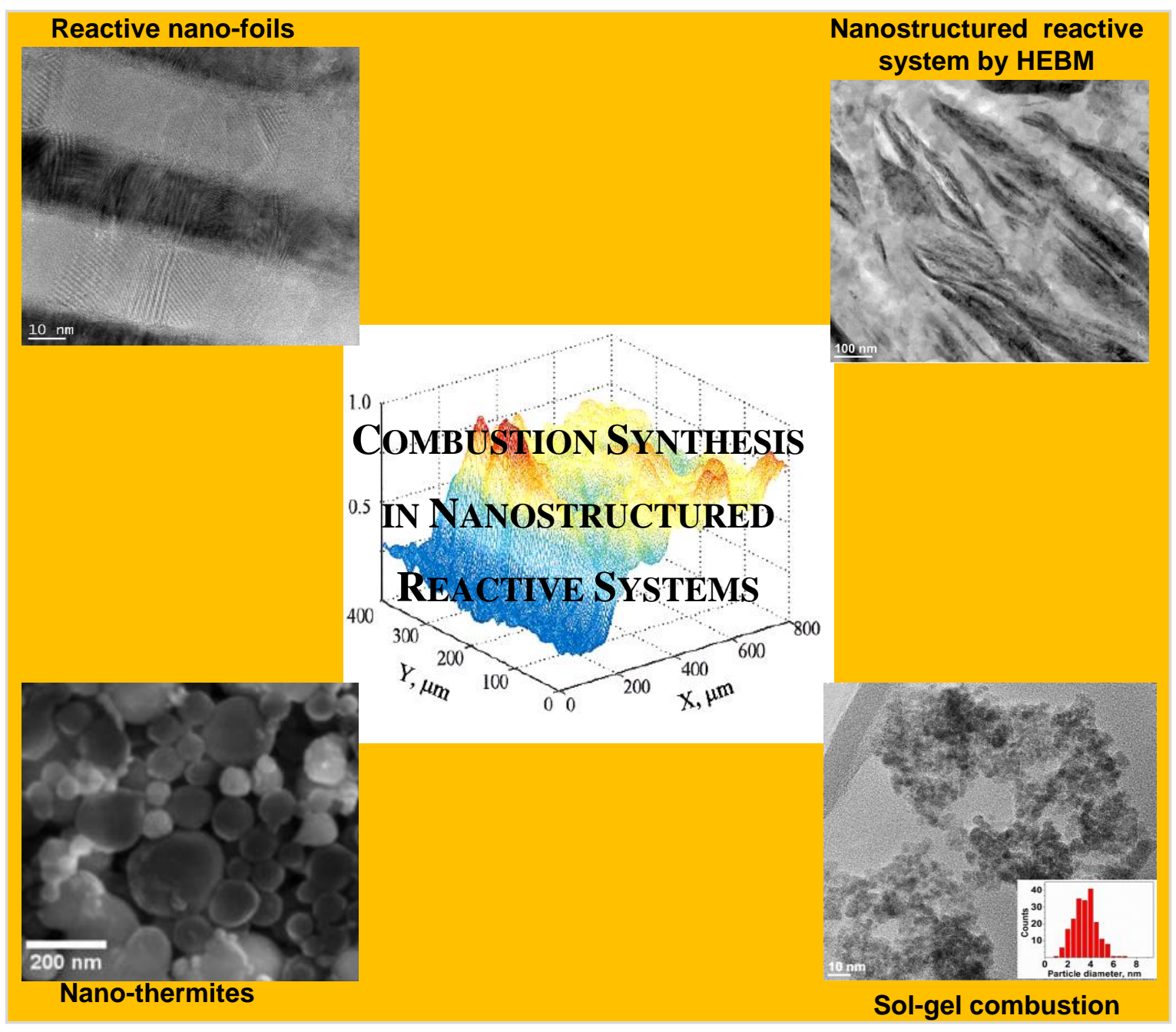




\title{
COMBUSTION SYNTHESIS IN NANOSTRUCTURED REACTIVE SYSTEMS
}

\author{
Alexander S. Mukasyan ${ }^{1,2^{*}}$ Alexander S. Rogachev ${ }^{2,3}$, Aruna Singanahally ThippaReddy ${ }^{4 *}$ \\ ${ }^{1}$ Department of Chemical and Biomolecular Engineering, University of Notre Dame, Notre \\ Dame, IN 46556, USA \\ ${ }^{2}$ Center of Functional Nano-Ceramics, National University of Science and Technology, \\ “MISIS”, Moscow 119049, Russia \\ ${ }^{3}$ Institute of Structural Macrokinetics and Materials Science, Russian Academy of Sciences, \\ Chernogolovka, 142432, Russia; \\ ${ }^{4}$ Surface Engineering Division, Council of Scientific and Industrial Research-National \\ Aerospace Laboratories, Bangalore 560 017, Karnataka, India
}

\begin{abstract}
New classes of reactive systems that are characterized by nano-scale heterogeneity and possess extremely high reactivity, as compared to that for similar reactive systems with micro-scale heterogeneity, have attracted a vast attention of many researchers. The recent developments and trends in combustion science towards such "nano" reactive media are presented. These systems include mechanically induced composite particles, sol-gels, super thermites and multilayer nanofoils. Various combustion-based applications of such nanostructured reactive systems are also discussed.
\end{abstract}

Key words: Combustion synthesis; Self-propagating high-temperature synthesis; Solution combustion; Sol-gel Auto combustion; Nanomaterials; Mechanical Activation; Super Thermites; Multilayer Reactive Nano-foils.

*Corresponding authors:

${ }^{4}$ E-mail: aruna_reddy@nal.res.in.

Tel.: +91 080 25086250; Fax: +91080252 10113.

1,2E-mail: amoukasi@nd.edu

Tel: +1-574-631-9825; Fax: +1-574-631-8366 


\section{Introduction}

Combustion synthesis (CS) or self-propagating high-temperature synthesis (SHS) is an attractive technique to synthesize a wide variety of advanced materials including powders and near-net shape products of ceramics, intermetallics, composites, and functionally graded materials. It is based on the ability of exothermic reactions to self-propagate in the heterogeneous media in the form of a glowing combustion wave producing solid materials with the desired microstructures, as well as physical and chemical properties. This method was discovered in the late sixties in the former Soviet Union [1,2]. The history of this discovery and development of its technological applications have been described in many books and reviews [3-7]. It is not necessary to reiterate these publications. We emphasize that the development of SHS has encountered-the hot enthusiasm and confidence for the enormous potential of this method, as well as the complete negation of the idea to use 'uncontrolled' combustion processes for the direct synthesis of materials. This is vividly described in the memoirs of A.G. Merzhanov, who is one of the founders of SHS [8].

It is worth noting that the combustion synthesis can be considered as a specific part of the powder metallurgy [3, 7]. Indeed, the traditional metallurgical methods based on the aluminothermic and magnesiothermic processes, have the basic features of combustion synthesis, i.e. local ignition, self-heating and self-acceleration of the reaction, propagation of the reaction wave, and formation of a useful product e.g. metal from ore. But the development of SHS has brought many new advances in the field. The search for gasless flammable compounds was first based on the use of iron-aluminium thermite heavily diluted by alumina in order to lower combustion temperature and suppress evaporation of the components. In these works the metallothermic process was studied using the methodology, which had been developed to study the combustion of gunpowder. This approach involves the organization of the plane front of the combustion wave propagating in one direction; recording the linear velocity of reaction front propagation; the study of the thermal structure of the wave; the identification of controlling stages and burning patterns. The novelty was already in the fact that the metallothermy was not studied as a process of metallurgy, but as a combustion process. Such approach led further to the discovery of other (non-thermite) type of combustible systems, which can be used for synthesis of materials. 
Another traditional powder-based method, which has similarity with SHS is the reactivebonding or reactive sintering, i.e. the consolidation process that involves the reaction between the components. However, during reactive sintering the technologies try to avoid the selfpropagation reaction at any point of the consolidated media, since it may result in non-uniformity of material's microstructure. In the above context, SHS can be considered as a limiting case of the reactive sintering. The CS technologies became feasible because the fundamental studies showed that if the physicochemical parameters of the medium, along with the instantaneous spatial distributions of temperature and concentration are known, one can calculate the combustion velocity and reaction rate throughout the reactive mixture. Thus, the SHS is a controllable, well-organized wavelike process, which leads to synthesis of materials with desired microstructures and properties.

Currently, scientists and engineers in many countries are involved in research and further development of combustion synthesis, and interesting theoretical, experimental, and technological results have been reported from various parts of the world. The number of recent publications on CS of metals, alloys, ceramics and composites, approaches ten thousand. Thus it is not possible to even briefly overview all of the latest achievements in this field. Our previous reviews on combustion synthesis were dedicated to specific aspects of this effective energy saving route to produce materials, which includes solution combustion synthesis of oxides [9], CS of nanopowders [10] and exothermic reaction waves in multilayer nano-foils [11] and other nano-systems [12]. This review places emphasis on material science aspects of nano-structured reactive systems.

Analysis of recent research papers on heterogeneous combustion has clearly revealed that the new classes of reactive systems, which are characterized by nanosized-scale and possess extremely high reactivity, as compared to similar reactive systems with micro-scale heterogeneity, has attracted the attention of researchers and has dominated R\&D. The present paper gives an overview of four classes of such reactive nano-media: (i) mechanically-structured composites; (ii) sol-gels; (iii) mixtures of metal and metal-oxide nanopowders, i.e., so-called, super thermite compositions and (iv) multilayer nano-foils. Research in each of these areas is being performed almost independently and cross-references are rare. However, a comparison of specific features of these phenomena is critical for fundamental understanding of interaction mechanisms in nanostructured combustible systems. The above systems are also attractive for a 
variety of advanced applications, such as: (i) synthesis of materials with pre-defined nanocrystalline structures and thus properties (mechanically-structured composites); (ii) synthesis of nanopowders including a wide range of catalysts (sol-gel systems); (iii) new generation of energetic materials (nano-thermite); (iv) joining/soldering of microelectronic components, refractory or dissimilar articles (multilayer reactive nano-foils). This review also attempts to discuss some of the above applications.

\section{Mechanically fabricated reactive microstructures and combustion synthesis}

High energy ball milling (HEBM) is a complex process that involves multiple deformations, fragmentation, cold welding, micro-diffusion, agglomeration, recrystallization, amorphization, micro-melting, crystallization and heterogeneous chemical reactions [13]. Two basic types of the HEBM process can be outlined [14]. First, is the so-called mechanical alloying process (MAP), which is used to produce novel alloys (phases) during HEBM. The second one is the mechanochemical processing (MCP) which describes the chemical reactions and phase transformations occurring in materials upon application of mechanical energy. MAP has resulted in the formation of large number of materials like $\mathrm{Al}-, \mathrm{Cu}$ - $\mathrm{Ni}$ - and $\mathrm{Fe}$ - based alloys reinforced with nano-oxide particles [15, 16], supersaturated crystalline solid solutions [17, 18], amorphous phases [19, 20], nanocrystalline solids [21, 22], solid solutions containing immiscible components [23], and other unique materials. The main feature of this process is the in-situ formation of new compounds during the mechanical treatment.

Mechanical activation (MA) can be distinguished from MCP by the fact that no chemical reactions or phase transformations take place during MA, however it influences the properties (e.g. reactivity) of the mechanically treated media. Recently, a large number of publications have been devoted to the so-called mechanically assisted self-propagating high-temperature synthesis (MASHS), which describes the synthesis of different materials by initiation of a combustion reaction in a heterogeneous mixture of powder precursors primarily subjected to high-energy ball milling $[12,24,25]$.

\subsection{Equipments used for mechanical alloying (MA)}

The spectrum of equipments used for production of nano-composite materials by MA is very broad and even a brief overview of the specific devices would go far beyond the scope of 
this review. Depending on the method, which is used to set the motion of milling balls, several types of devices are recognized. In an attritor mill, the mixing tank is stationary, and the balls are moved by the rotating stirrer (mixer) inserted into the vessel. In a rotary ball mill, the cylindrical vessel is lying on the drive rollers, and the motion of the balls (inside the jar) is caused by rotation of the vessel around its horizontal axis. The planetary ball mill combines rotation of two or more jars around their own axes with rotation around its common general axis, similar to the revolution of planets around the Sun. Comparative considerations of various milling devices has been made in several reviews and books [26-29].

The most widely used methods for mechanical treatment of reactive materials are rotary and planetary ball mills. Trajectories of the balls inside the vessel have also been the subject of numerous theoretical and experimental studies. Depending on the rotation speed, friction force, size of the balls and other parameters, the balls can: (i) roll or slide along the inner surface of the jar's wall; (ii) fall down inside the vessel and (iii) make periodic circular or chaotic swirling motions. To show the correlations between the rotation regimes and trajectories of the balls for different mills, special parametric maps have been plotted (cf. [29]). However, there are no direct experimental data on the real ball behavior during milling. Moreover, the limited available observations often contradict the theoretical predictions. Recently, direct video recording of the motion of balls has been made by using a video camera installed on the rotating "sun wheel" above the jar, covered with a transparent lid [30]. Unexpectedly, analysis of the recorded images showed essentially only rolling and sliding of the balls for all operational parametric regions for the considered planetary mill. No free falling ball regime was observed. These results indicate that friction and slip may play much more significant role during HEBM than it was assumed based on theoretical models.

During HEBM, the modification of the micro- and crystal structures of the heterogeneous powder mixtures that occurs may also depend on the intrinsic values of temperature and pressure in the zones of "ball-ball" or "ball-wall" contacts. The calorimetric measurements of the balls and the vial immediately after the milling procedure indicated that the temperature of the steel balls (7-9 $\mathrm{mm}$ in diameter) reaches as high as $873 \mathrm{~K}$ after 10 minutes of milling [31]. This high temperature was detected for the most intense regime (balls velocity $8.24 \mathrm{~m} / \mathrm{s}$ ) of the AGO-2 planetary ball mill, in an inert atmosphere ( $0.1 \mathrm{MPa}$ of $\mathrm{Ar}$ ) and without any powder in the vial. Other measurements using wireless temperature and gas pressure monitoring system in a Fritsch 
Pulverisette P6 planetary ball mill have shown much lower vial temperatures ( $\sim 313 \mathrm{~K})$ [32]. Thus for the proper comparison of the results reported in literature, it is critical to know the HEBM parametric regions, which define the mechanism of the ball-powder interaction.

\subsection{Fundamentals of MASHS}

In general, it has been established that high-energy ball milling of reactive mixtures significantly enhance their reactivity $[12,24,25,33,34]$. The reported effects of HEBM on materials synthesis by use of the combustion approach can be classified as follows:

(a) Expansion of the combustion limits: The HEBM of weakly exothermic compositions, under conventional conditions, does not permit the combustion reaction mode and leads to the possibility of self-sustained reaction regime in such systems; (b) After HEBM the ignition temperature of the system can be several hundred degrees lower, as compared to conventional mixtures; (c) Typically solely solid state reactions are responsible for the reaction initiation in mechanically activated systems; (d) The mechanical activation of green mixtures results in an increased combustion velocity (even for mixtures which react without HEBM, e.g. Ni-Al); (e) In some cases, the combustion temperature of mechanically activated mixtures turns out to be higher than that for similar conventional mixtures. Differential thermal analysis (DTA)/calorimetric analysis reveal an increase in overall heat released during the reaction of such mechanically pretreated systems. However, the combustion temperature and thermal effects of the activated mixtures never exceed the thermodynamically pre-determined adiabatic temperature and enthalpy of the reaction; (f) Finally, it was shown that the microstructure of the products and materials synthesized from mechanically activated mixtures differs from those obtained by non-activated CS.

\subsection{Hypotheses and mechanisms}

Several hypotheses have been suggested in literature to explain the above effects of HEBM on the reactivity of heterogeneous powder mixtures. These hypotheses were recently analyzed for the Ni-Al system [35]. The most important ones are: (i) decreasing apparent activation energy by the magnitude of the energy stored into reactants during MA; (ii) increasing atomic mobility due to a high concentration of grain boundaries and defects created by plastic deformation; (iii) formation of non-equilibrium meta-stable phases; (iv) increase of the contact (interfacial) surface 
area between reactants; (v) formation of freshly formed reactive surfaces and (vi) nano-scale mixing of the reagents.

Thus two groups of effects can be outlined based on energy and microstructure. The energybased mechanism assumes the accumulation of some additional energy by the crystal lattice of particles undergoing mechanical activation. However, the excess of enthalpy, stored by the lattice defects and lattice strain during mechanical treatment, usually does not exceed a few $\mathrm{kJ} / \mathrm{mole}$ even for nanometer-scale grain size $[35,36]$. This value is much lower than the enthalpy of formation from the elements (e.g., for $\mathrm{Ti}+\mathrm{C}=\mathrm{TiC}, \Delta \mathrm{H}=209 \mathrm{~kJ} / \mathrm{mole}$ ). Therefore, the energy accumulated by crystal lattices can hardly be responsible for increasing the combustion temperature and the other above mentioned effects.

Microstructure-based explanations assume that acceleration of combustion synthesis occur due to a decrease in the effective reactant size (milling, multiple ductile deformations) and an increase of contact surface area between the reactants. Through high resolution electron microscopy $[35,37,38]$, it was shown that short term $(<10 \mathrm{~min})$ HEBM not only increases the surface area of contacts between reagents by thinning and folding of the materials, but more importantly, creates regions where nanometric $\mathrm{Ni}$ is embedded in an $\mathrm{Al}$ matrix, without oxide barriers. The appearance and development of nanometrically mixed region is attributed to the significant enhancement of the reactivity of the system.

Assuming Arrhenius approximation, the kinetics of reaction between $\mathrm{Ni}$ and $\mathrm{Al}$, can be presented as follows:

$$
\frac{\partial \eta}{\partial t}=F(\eta, T)=A(T) \cdot S_{c} \cdot e^{-\frac{E_{e f}}{R T}} \cdot \Phi(\eta)
$$

where $\eta$ - degree of conversion; $S_{c}-$ contact area between the reactants; $E_{\text {ef }}-$ effective or apparent activation energy of reaction; $A(T)$ - pre-exponent function and $\Phi(\eta)$ - function which accounts the dependence of reaction rate on the order of the reaction $(\eta)$. From this equation it can be concluded that the reaction takes place at any temperature, $S_{c}$ and $E_{e f}$ values. However, the important issue is the rate of heat evolution. If the rate of heat release is lower than that of the heat loss (e.g. through conduction and convection) from the reaction volume, the self-sustained process cannot be accomplished (Figure 1: $\mathrm{T}<\mathrm{T}_{\mathrm{eu}}$ ). Before HEBM, reactants have extremely small, "point"-type contact areas. Also it is well known that metal particles, especially Al, have oxide layers on their surfaces, which significantly increases the energy barrier for the metal- 
metal reaction $\left(\mathrm{E}_{\mathrm{ef}}\right)$. For these reasons the ignition temperature in the $\mathrm{Ni}-\mathrm{Al}$ system is equal to or above the eutectics $(912 \mathrm{~K})$ [39]. When the melt is formed, the oxygen film ruptures and $\mathrm{Al}$ spreads along the surface of $\mathrm{Ni}$ particle increasing $\mathrm{S}_{\mathrm{c}}$. Also liquid-solid reactions are characterized by lower activation energies as compared to solid-solid ones. Thus the rate of heat evolution ( $\mathrm{q}+$ ) abruptly increases at $\mathrm{T}=\mathrm{T}_{\text {eu }}$ and becomes higher than the heat loss rate (q-), i.e. self-sustained process initiates (Figure 1: $\mathrm{T}>\mathrm{T}_{\mathrm{eu}}$ ).

\subsection{Nanostructured reactive systems prepared by MASHS}

During HEBM of the $\mathrm{Ni}+\mathrm{Al}$ powder mixture (Figure 2a) in an inert atmosphere, composite particles of both $\mathrm{Ni}$ and $\mathrm{Al}$ phases are formed (Figure $2 \mathrm{~b} \& 2 \mathrm{c}$ ). It is more important that these phases possess high contact surface area and are devoid of oxygen [35]. This leads to a significant decrease of reaction activation energy, which was experimentally confirmed both by TGA/DTA and electro-thermal explosion methods $[40,41]$ and thus in some sense, a mechanical activation process (MAP) of the reaction takes place. However, one more microstructural aspect, which shows the formation of zones with the characteristic size of $\mathrm{Ni}$ and $\mathrm{Al}$ phases in nanometer range is important (Figure 2d) [35,37,38]. It is clear that in such nano-mixtures, the reactive diffusion on the scale of several nanometers leads to a significant degree of conversion, resulting in rapid heat release rates even at relatively low temperatures. Dependences of ignition temperature $\left(\mathrm{T}_{\mathrm{i}}\right)$ as a function of content of nano-scale mixed areas in composite particles are shown in Figure 3 [38]. It has been theoretically predicted that for $E_{\text {eff }} 250 \mathrm{~kJ} / \mathrm{mol}, \mathrm{T}_{\mathrm{i}}$ can be as low as $500 \mathrm{~K}$ and this value was confirmed experimentally [40, 42, 43]. Thus the self-sustained rapid reaction of such composite particles occurs at temperatures well below the melting point of Al for which solid state mass-transfer is responsible. This result takes us back to the basic concept of "solid flame" suggested by A.G. Merzhanov in 1967 [4], which claim that a rapid combustion wave may propagate along the heterogeneous reactive media due to the solely solid state interactions.

The above discoveries are important for controlling the conditions for combustion synthesis of NiAl based intermetallics. Specifically, an extremely rapid reaction in the "nanomixed" composite particles, which is initiated at a temperature well below the melting point of $\mathrm{Al}$, leads to the fact that the melting process does not influence the microstructure of the media (Compare Fig $4 \mathrm{a}$ and b) [37, 42]. Thus the microstructure of the CS material "mimics" the 
microstructure of green samples. The latter allows one to control the material structure and thus properties, by pre-designing of the microstructure the final material by controlling that of the "green" sample.

Similar results were obtained for the titanium-graphite system [44]. It was shown that short term (1-7 min) HEBM converts the initial powder mixture (Fig.5a) to the composite particles with a size range of $1-5 \mu \mathrm{m}$ (Fig.5b). The cross section of a typical Ti/C composite particle is shown in Fig 5c. It can be seen that it consists of sub-micron layers of Ti and C. XRD and TEM analysis revealed the amorphous nature of the carbon layers thus confirming the formation of nanoscale structures using HEBM. Such nano-mixed particles possess extremely low reaction onset-temperature $(\sim 500-600 \mathrm{~K})$, which is 3 times lower than that for the conventional powder mixture. It is more important that combustion synthesis in such MA mixtures leads to the formation of sub-micron titanium carbide powders with an average particle size of $\sim 100 \mathrm{~nm}$ (Fig.5d) and a specific surface area of $25 \mathrm{~m}^{2} / \mathrm{g}$. The mechanism for the formation of such nano-powder is not yet clear. It is possible that the disintegration of micron size $(1-5 \mu \mathrm{m})$ composite particles takes place in the combustion wave due to the intensive evolution of hydrogen initially adsorbed on titanium layers. Similarly, nano-particles of other metals from groups IV and V may be synthesized by MASHS approach.

Another example of using MASHS is the combustion synthesis of sub-micron $\mathrm{SiC}$ powders. From the viewpoint of chemical nature, the gasless combustion reaction from the elemental powders in $\mathrm{Si}-\mathrm{C}$ system is characterized by the following equation:

$$
\mathrm{Si}^{(\mathrm{s}, 1)}+\mathrm{C}^{(\mathrm{s})} \rightarrow \mathrm{SiC}^{(\mathrm{s})} ; \Delta \mathrm{H}_{273}=73 \mathrm{~kJ} / \mathrm{mol}
$$

It can be seen that the reaction (2) has a moderate enthalpy of product formation (e.g. compared with $\Delta \mathrm{H}_{273}=230 \mathrm{~kJ} / \mathrm{mol}$ for Ti-C system) and is thus characterized by a relatively low adiabatic combustion temperature $\left(\mathrm{T}_{\mathrm{ad}}=1873 \mathrm{~K}\right.$; while for the $\mathrm{Ti}+\mathrm{C}$ reaction $\left.\mathrm{T}_{\mathrm{ad}}=3290 \mathrm{~K}\right)$. This feature implies that it is not easy to accomplish a self-sustained SHS process in this system.

Several approaches have been developed to enhance the reactivity of the $\mathrm{Si}-\mathrm{C}$ system, which can be subdivided into four categories [45]:

- $\quad$ SHS with preliminary preheating of the reactive media: Pampuch et al. [46] showed that a uniform preheating of the stoichiometric $\mathrm{Si}+\mathrm{C}$ mixture in a flow of argon gas leads to selfignition of the heterogeneous media at a temperature of $\sim 1600 \mathrm{~K}$ with the formation of $\beta$-SiC powders with a morphology similar to that of the initial carbon; 
- SHS with an additional electrical field: This method proposed by Yamada et al, [47] was used for the synthesis of different SiC-based ceramics (see also [48-50]);

- Chemically activated SHS: This method was pioneered by Nersisyan et al., [51] wherein chemical additives like polytetrafluoroethylene (PTFE), which allows gas-phase reaction routes, are added as activation agents for $\mathrm{Si}+\mathrm{C}$ reaction

- SHS synthesis in a reactive atmosphere: for example, in nitrogen or air, since the reaction between silicon and nitrogen is much more exothermic as compared to $\mathrm{Si}+\mathrm{C}$ one [52].

The first method for CS of silicon carbide, which involved HEBM consists of the following steps [53]: (i) addition of a small amount (1-3 wt\%) of $\mathrm{NH}_{4} \mathrm{Cl}$ and $\mathrm{PVC}$ into the $\mathrm{Si}+$ C powder mixture; (ii) mechanical activation of the mixtures through HEBM (2-12 h); (iii) preheating the mixture to a desired temperature $(1200-1500 \mathrm{~K})$ and maintaining it at this temperature until the reaction self-initiates. Fine $\beta$-SiC powder with a specific surface area of 4.5 $\mathrm{m}^{2} / \mathrm{g}$ and particle size $<5 \mu \mathrm{m}$ were synthesized. However, the above method involves the combination of several approaches, i.e., mechanical and chemical activations, as well as preheating, which makes it complex and requires high external energy consumption. The mechanically activated pure $\mathrm{Si}+\mathrm{C}$ mixtures (without any additives) were burned in air to synthesize nano-SiC powder. In the SHS mode [54] it was shown that after $4 \mathrm{~h}$ of HEBM the mixture can be ignited in air at 1 atm. During the initial stages, the surface reaction between oxygen and silicon leads to the formation of a relatively thin layer of $\mathrm{SiO}_{2}$ phase, which simultaneously preheats the bulk media. This reaction is followed by a combustion wave due to the reaction between silicon and carbon with the formation of $\beta$-SiC and a small amount of $\mathrm{Si}_{2} \mathrm{~N}_{2} \mathrm{O}$ phases. Again, the combination of relatively long-term HEBM and oxidation/nitridation reactions were used to produce ceramic powder in SHS mode.

It was only recently $[55,56]$ demonstrated that short-term (15 minutes) HEBM allows the direct combustion synthesis of silicon carbide powders in an inert (argon) atmosphere without the addition of any chemical activators. The initial micron sized silicon and graphite powder mixtures (Fig.6a) after 15 minutes of milling transforms to the nano-sized composite particles (Fig.6b), which consist of amorphous carbon and crystalline silicon. Self-sustained reaction can be accomplished in such nano-media in an argon atmosphere that propagates with velocity of $\sim 5$ $\mathrm{mm} / \mathrm{s}$ and has a maximum temperature of $1800 \mathrm{~K}$. Under optimum conditions, the synthesis of 
sub-micron (50-200 nm) SiC powder with a specific BET surface area of $\sim 20 \mathrm{~m}^{2} / \mathrm{g}$ can be accomplished in the self-sustained reaction (Figure $6 \mathrm{c}, \mathrm{d}$ ).

All the above examples demonstrate MASHS to be a powerful tool for the synthesis of nano-materials. Based on the understanding of fundamental processes, which occur during short term high energy ball milling, one may produce powders of desired microstructure and properties. High energy ball milling allows formation of composite particles with reagents mixed on the nanoscale level. It is worth noting that HEBM being attractive method for the synthesis of nano-powders cannot be directly used for bulk materials production and to achieve this goal additional energy consuming sintering stage is required. The combination of HEBM + CS permits the formation of nano-structured bulk materials. Direct synthesis of such materials and net shape articles by using MASHS approach is a promising next step in development of this scientific endeavor.

\section{Sol-gel combustion synthesis}

With the advent of solution combustion synthesis (SCS) in 1986, a large number of research efforts have been focused towards the preparation of technologically important materials, mostly oxides, with improved properties [8, 9]. In solution combustion synthesis, aqueous reactive solutions are used, where the precursors are mixed essentially on the molecular level. The versatility of this process has been demonstrated by the use of different fuels and oxidizers, varying the oxidizer/fuel $(\mathrm{O} / \mathrm{F})$ ratio and ignition sources, as well as combination of various synthesis approaches [57]. Among these, sol-gel combustion is an important modification of SCS that has shown the potential of yielding not only oxides but also metals, alloys and metal foams [58]. The sol-gel combustion (SGC) process, as the name indicates, is a unique combination of chemical gelation and combustion processes. Most of the used systems are highly exothermic and hence, due to the release of large amounts of heat, the phase formation of the final product occurs in the high temperature reaction front. Thus the compounds synthesized by SGC are typically well crystallized and essentially no post-combustion calcination is required. The SGC synthesis of ceramic oxides offers advantages such as high purity and good homogeneity [59]. Recent developments and trends in SGC method for the synthesis of nanosized spinel ferrites are briefly discussed and critically analyzed in a recent review [60]. More process details including the recent advancements in SGC are discussed below. 


\subsection{Sol-gel combustion modes}

In both the SCS and SGC methods the precursors used are usually metal nitrates (oxidizers) and nitrogen containing organic substances such as urea, glycine, and citric acid (fuels). The main differences lie in the fact that in the sol-gel combustion method, the $\mathrm{pH}$ of the redox mixture is typically controlled by the addition of a weak base (e.g. ammonia) and the aqueous solution is dried to evaporate most of the water and forms a highly viscous reactive gel. Similarly to conventional combustion synthesis systems, in reactive gels, the combustion may proceed in different modes. In self-sustained propagation mode, the reaction is locally initiated and the combustion wave rapidly propagates through the gel converting the media to a valuable solid product. The temperature of the wave front in SGC is typically higher than that for conventional SCS. During the volume combustion synthesis mode, the bulk of the gel is heated uniformly in a controlled manner until the reaction occurs almost simultaneously throughout its volume. In both modes, the combustion reaction changes the gel to homogenous, highly pure nanopowders or highly porous foams $[59,60]$.

Let us consider an example for the specifics of self-sustained combustion mode, which allows more for controllable synthesis conditions as compared to those for volume combustion mode. Figure 7 shows the examples of temperature-time histories for the synthesis process in several nickel nitrate-glycine gels, which were formed at different gelation temperatures $\left(\mathrm{T}_{\mathrm{g}}\right)$ [61]. It can be seen that that the maximum combustion temperature increases from 900 to $1150^{\circ} \mathrm{C}$ with increase of $\mathrm{T}_{\mathrm{g}}$ from $30-90^{\circ} \mathrm{C}$. It is worth noting that the propagation velocity of the reaction front, $\mathrm{U}_{\mathrm{c}}$, also increases from $0.25 \mathrm{~cm} / \mathrm{s}\left(\mathrm{T}_{\mathrm{g}}=300 \mathrm{~K}\right)$ to $0.45 \mathrm{~cm} / \mathrm{s}\left(\mathrm{T}_{\mathrm{g}}=360 \mathrm{~K}\right)$. The data demonstrates that gel preparation parameters are critical to control the synthesis conditions.

Detailed IR image analysis of the combustion wave (Figure 8a) in reactive gels indicates that the total width of combustion wave does not exceed $1 \mathrm{~mm}$. A magnified view of the reaction zone (Figure 8b) and corresponding two-dimensional temperature distribution map (Figure 8c) clearly suggests three distinctive zones in the combustion wave. Zones 1 and 2, are the so-called preheating zone, in which there is no chemical reaction. Intensive evaporation of bounded water takes place in zone 1, which leads to the formation of water free reaction mixture in zone 2 . The exothermic reaction begins on the boundary between zones 2 and 3. The estimated width of the chemical reaction zone 3 is $\sim 0.5 \mathrm{~mm}$. Taking into account that $\mathrm{U}_{\mathrm{c}}$ in this system in the range 0.1 - 

in the reaction zone and rapid cooling $\left(>10^{2} \mathrm{~K} / \mathrm{s}\right)$ outlines the uniqueness of the synthesis conditions.

\subsection{Fuels, additives and their roles}

Organic compounds containing chelating ligands like carboxylate groups (citric acid, glycine) or aliphatic amine groups (hydrazides, glycine) are the most widely used fuels for the synthesis of metallic oxides. These agents can effectively facilitate the chelation of metal ions of varying ionic sizes, which helps in preventing their selective precipitation and in turn maintain compositional homogeneity among the constituents and also serve as a fuel in the combustion reaction. In SGC method, citric acid is the most widely used fuel and has been mostly utilized for producing a large variety of ferrites [60]. It is relatively inexpensive and a more effective complexing agent as compared to glycine, urea, and hydrazide based fuels. Nitrate salts are the preferred precursors, as they are both water-soluble and are low temperature oxidizer sources for synthesis [62].

It is possible to control the system stability, microstructure, reaction temperature, the rate and volume of gases generated by choosing appropriate complexing agent, oxidizer to fuel ratio $(\mathrm{O} / \mathrm{F}, \Phi)$, chemical additives, heating source and atmosphere. Ammonia increases metal cation chelation with citric acid, which could help to control the oxygen balance and develop a porous 3D network in nitrate-citrate aerogels. Excess or deficient fuel is characterized by a lower combustion rate and combustion temperature, this in turn reduces the possibility of particle growth and sintering at the same time. However, the product obtained may have lower crystallinity and phase purity compared to the stoichiometric compositions. The addition of an appropriate amount of $\mathrm{NH}_{4} \mathrm{NO}_{3}$ to the reactant mixture will compensate for the loss of $\mathrm{NO}_{3}$ during the dehydration process [63]. With an increasing $\mathrm{pH}$ value, crystallite size increases due to higher combustion temperature. Ethylene glycol acts as a gelating agent as it forms an organic ester by forming bonds with the complexant $[64,65]$. Polymerization is facilitated by heating and results in the formation of a homogeneous polymer network with metal ions uniformly distributed throughout the organic matrix. 


\subsection{Influence of atmosphere and heating source}

The ambient atmosphere can influence the nature of combustion reactions. For e.g., the mass-changing rate in nitrogen will be nearly twice that compared to an air atmosphere. This is due to the supply of additional nitrogen to the gel and formation of $\mathrm{NO}_{3}$ ions acting as oxidants and the reaction could be restrained in nitrogen [66]. Also, the temperature for the spinel ferrite formation after the combustion reaction is lower in air than in nitrogen [67]. The reaction rate is slower and temperature higher when ignition is induced in a muffle furnace due to differences in oxygen availability. Employing a hot plate during the combustion reaction of ferrites yielded pure spinel type structures, while products formed in the muffle furnace revealed hematite as the secondary phase. Also, powders obtained on a hot plate were characterized by lower particle and agglomerate sizes and higher specific surface area. Heat is generated from inside the gel in case of microwave heating, in contrast to conventional heating methods in which heating proceeds from the surface [68].

\subsection{Oxidizer to fuel ratio}

Metal, alloy or cermet foams that can be used for a wide range of potential applications can be prepared by SGC method by changing the oxidizer to fuel ratio $(\Phi)$ values [69-71]. Nearstoichiometric conditions produced a vigorous reaction, yielding a mixture of fine cermet-type foam consisting of $\mathrm{Ni}$ and $\mathrm{NiO}$ phases. For $\Phi>1.25$ i.e., fuel-rich conditions, the reaction progressed much more slowly with a flame front moving through the reactant solution, leaving behind pure Ni foam, while the material synthesized using $\Phi<0.8$ value was pure metal oxide. These findings are important as they indicate that high purity metal or cermet foams can be obtained through SGC, even when the reaction takes place in an open container, simply by selecting a proper fuel to oxidizer ratio $(\Phi)$. However, it has to be noted that the critical $\Phi_{\text {cr }}$ values, above which pure metal can be obtained are different for different systems, e.g., for $\mathrm{Cu}$, $\Phi_{\mathrm{cr}}=2.5$; and for $90 \% \mathrm{Ni}-10 \% \mathrm{Cu}$ alloy $\Phi_{\mathrm{cr}}=1.5[69]$.

Specific reaction pathways have been suggested by Kumar et al., to describe the metallic phase formation during the synthesis of nanopowders by SGC approach [70, 71]. It is proposed that the exothermic reaction between $\mathrm{NH}_{3}$ and $\mathrm{HNO}_{3}$ species formed during the decomposition of glycine and nickel nitrate acts as the source of energy required to achieve the self-sustained 
reaction regime. A thermodynamic analysis of the combustion synthesis reaction indicates that increasing glycine concentration leads to establishing a hydrogen rich reducing environment in the combustion wave that in turn results in the reduction of metal oxide phases and the formation of pure metals or/and metal alloys.

It can be concluded from the above that the sol-gel combustion is a versatile technique for the preparation of porous nanostructured materials that possess high surface area, ultralow density and high-strength-to-weight ratio.

\subsection{SGC synthesized materials}

Nanoporous metal foams (NMFs) with high surface area have been a long sought-after class of materials as they can find applications in high power-density batteries, hydrogen storage, high-field magnets, and production of fuel cell anodes and hybrid supercapacitors. An overview of newly developed synthetic strategies for producing NMFs along with an in-depth discussion of combustion synthesis as a versatile and scalable approach for the preparation of nanoporous, nanostructured metal foams was presented by Tappan et al. [72, 73]. The technique for producing metal nano foams using combustion synthesis was discovered during the characterization of a class of energetic salts and transition-metal complexes being developed at Los Alamos. Chavez et al. had synthesized the alkali- and alkaline-earth-metal salts and copper complexes of several members of the tetrazole family, primarily those of bistetrazole (BT) and bistetrazolamine (BTA) [74]. KlapÖtke et al. has since explored these and other related complexes in depth $[75,76]$. As energetic additives to pyrotechnics, these compounds produce color through excitation of metal atoms released during combustion, generating primarily only $\mathrm{N}_{2}$ and $\mathrm{CO}_{2}$ gas as by-products. Hunt et al. [77] demonstrated a different type of combustion process for preparing $\mathrm{NiAl}$ intermetallic foams by passivating nanometer-sized $\mathrm{Al}$ particles with fluorinated organic ligands $\left(\mathrm{C}_{13} \mathrm{~F}_{27} \mathrm{COOH}\right)$ that double as gasifying agents. These foams could potentially serve as useful precursors to nanoporous Raney Ni foams (Raney Ni foam is a fine grained solid composed of $\mathrm{Ni}$ derived from $\mathrm{Ni}-\mathrm{Al}$ prepared by leaching the $\mathrm{Al}$ using concentrated sodium hydroxide). Combustion synthesis of metal foams with nanoporous structures was first demonstrated by Tappan et al by use of yet another combustion synthesis technique involving metal complexes containing the high-nitrogen ligand ammonium tris(bi(tetrazolato)amine)ferrate(III) (FeBTA) [72]. In this approach, metal bistetrazolamine 
(MBTA) complexes were synthesized, pressed into pellets, and ignited under an inert atmosphere. The result is the formation of metal foams (Fig. 9) with pores primarily ranging from a few nanometers to several microns in diameter. The materials produced through combustion synthesis of MBTA complexes are referred to as "nanofoams", as they are not only nanoporous but also intrinsically nanostructured-a property that differentiates their potential technological applications markedly from those of macroporous foams.

Insight into the assembly mechanism underlying the formation of metal nano-foams has been obtained by analyzing the heats of formation and calculated adiabatic flame temperature which, suggests that melting does not occur during formation of the foam and that the foam assembly occurs by rapid sintering and/or localized surface melting in a highly non-equilibrated state [72]. Thus control over wave propagation is important for enabling the formation of foams over powders, which result if the combustion wave propagates too vigorously.

Nanoporous iron metal foams (Fig.10) were also synthesized by an improved SGC method using citric acid and ethanol by adjusting the $\mathrm{pH}$ to $\sim 5-6$ by ammonia $[78,79]$. From the photographs shown it can be seen that the volume of the synthesized sample is about ten times larger than that of the initial sample. The synthesis method is inexpensive and very convenient, and the obtained iron foams present quite large saturation magnetization at room temperature. The developed ferromagnetic porous iron foams may find wide applications in the fields of catalysis, fuel cells, hydrogen storage, unique insulation, and electromagnetic absorption.

The reduction ability of the SGC process was improved by the addition of a suitable amount of ethanol during the preparation of the sol; thus, the metal ion could be reduced from its dried gel by the improved SGC process. This makes the synthesis of metal foams in the forefront of materials science. Transition metal foams of nickel, copper, cobalt, and $\mathrm{Ni}-\mathrm{Cu}$ and $\mathrm{Ni}-\mathrm{Co}$ alloys have been synthesized with the controlled combustion method by adjusting the $\mathrm{O} / \mathrm{F}$ ratio. $\mathrm{Cu}, \mathrm{Ni}$ and $\mathrm{CuNi}$ alloy nanoparticles prepared using citric acid and ethylene glycol were sintered at $800{ }^{\circ} \mathrm{C}$ and their resistivity values were equal to or two times higher than that of the bulk phase Constantan $\left(\mathrm{CuNi}_{44} \mathrm{Mn}_{1}, \rho=5 \cdot 10^{-7} \Omega \mathrm{m}\right)$ and about 7-13 times lower than the earlier $7.3 \times 10^{-6} \Omega \mathrm{m}$ reported for Ni-Cu thick film [79]. Printed van der Pauw $\mathrm{Cu}-\mathrm{Ni}$ structures which is commonly used in resistive applications with a thickness of $1-4 \mu \mathrm{m}$ were deposited on a Siwafer from ethylene glycolic dispersions $\left(\mathrm{c}=25 \mathrm{~g} / 100 \mathrm{~cm}^{3}\right)$ of $\mathrm{CuNi}$ alloy nanoparticles by means of Aerosol Jet ${ }^{\circledR}$ printing (Fig. 11). From an application perspective, these results 
demonstrate that SGC synthesized alloy nanoparticles are suitable for the preparation of printed conductive structures by AerosolJet ${ }^{\circledR}$ printing.

Nickel-based catalysts are used in many industrial processes including hydrogenation reactions, reforming reactions, Fischer-Tropsch synthesis, hydrogen production, ethanol decomposition etc. More specifically, as research interest on fuel cell technology increases, the need for a hydrogen supply is also rising. The solution combustion method was used to prepare $\mathrm{Ni}$ catalysts supported on $\gamma-\mathrm{Al}_{2} \mathrm{O}_{3}$ pellets with controlled metal distribution. It was shown that impregnation time of the SCS solution onto the pellet determines the distribution of the Ni in the pellet which in turn affects the catalyst selectivity towards hydrogen production during ethanol decomposition [80].

Shinde and Madras have reported the combustion synthesis and catalytic activity of ionic substituted $\mathrm{Cu}$ catalysts in the reducible supports $\left(\mathrm{SnO}_{2}, \mathrm{CeO}_{2}\right)$ and non-reducible supports $\left(\mathrm{Al}_{2} \mathrm{O}_{3}\right.$ and $\left.\mathrm{ZrO}_{2}\right)$ [81]. An important feature of this synthesis is that a tin oxalate precursor was used as an oxidizer. 5 at $\% \mathrm{Cu}$ impregnated over combustion synthesized $\mathrm{SnO}_{2}$ and $\mathrm{ZrO}_{2}$ were prepared by reducing $\mathrm{Cu}\left(\mathrm{NO}_{3}\right)_{2}$ dispersed over the oxide supports by hydrazine. The resulting dried sample was reduced in $\mathrm{H}_{2}$ at $350{ }^{\circ} \mathrm{C}$ for $3 \mathrm{~h}$ and used for catalytic studies. All compounds showed high activity for the catalytic combustion of $\mathrm{H}_{2}$ and $100 \%$ conversion of $\mathrm{H}_{2}$ was obtained below $480{ }^{\circ} \mathrm{C}$.

The zirconia and doped zirconia was prepared by SGC technique using zirconium(IV) nbutoxide, 1-butanol and glacial acetic acid as precursors [82]. The three catalyst powders, were tested in a catalytic-bed reactor for the combustion of trichloroethylene, and the mass-transfer effects were evaluated and found to be negligible due to the size of the particles. The average overall mass-transfer coefficients were conveniently estimated by the Yoshida correlation. The kinetic studies showed that the $\mathrm{ZLF}\left(\mathrm{ZrO}_{2}\right.$ doped with $\mathrm{La}$ and $\mathrm{Fe}$ ) catalyst was more active than the $\mathrm{Z}\left(\mathrm{ZrO}_{2}\right)$, and $\mathrm{ZLMF}\left(\mathrm{ZrO}_{2}\right.$ doped with $\mathrm{La}, \mathrm{Mn}$ and $\left.\mathrm{Fe}\right)$ catalyst. The catalysts presented low specific areas but good activity; however, the relatively large cavity sizes (Fig. 12) facilitated the transport of trichloroethylene and combustion products through the porous structure of $\mathrm{ZrO}_{2}$ in the catalytic tests.

The performance of dye-sensitized solar cells developed using $\mathrm{TiO}_{2}$ powders synthesized by the sol-gel combustion hybrid method using titanium isopropoxide, isopropanol and acetylene carbon black and calcined at $500{ }^{\circ} \mathrm{C}$ and $600{ }^{\circ} \mathrm{C}$, was evaluated and showed a solar conversion 
efficiency of 5.02\% [83]. Layered $\mathrm{LiMn}_{0.4} \mathrm{Ni}_{0.4} \mathrm{Co}_{0.2} \mathrm{O}_{2}$ and $\mathrm{LiMn}_{1 / 3} \mathrm{Ni}_{1 / 3} \mathrm{Co}_{1 / 3} \mathrm{O}_{2}$ compounds suitable for lithium ion batteries were synthesized using corn starch-assisted SGC methods [84]. $\mathrm{LiMn}_{1 / 3} \mathrm{Ni}_{1 / 3} \mathrm{Co}_{1 / 3} \mathrm{O}_{2}$ cathode exhibited an appreciable capacity of $176 \mathrm{mAh} \mathrm{g} \mathrm{g}^{-1}$, excellent capacity retention of $93 \%$ up to 100 cycles and susceptible to rate capability test up to $1 \mathrm{C}$ rate, thus qualifying the same for high-capacity and high rate lithium battery applications.

Recently the SGC method has successfully yielded one-dimensional (1D) and twodimensional (2D) nano-structures of advanced spinel ferrite [60]. It has been shown that an array of highly ordered hydroxyapatite (HAP) nanotubes can be synthesized by the SGC method with a porous anodic aluminum oxide (AAO) template [85]. All the above applications and range of materials discussed above proves the versatility of the SGC process.

\section{Nanothermites}

Thermite reactions, which are used to purify ores of some metals, are called the thermite process, or aluminothermic or magnesium-thermic reactions and have been discovered almost two centuries ago. Such reactions have been adapted and used to obtain even pure uranium [86]. Thermite by common definition is a pyrotechnic heterogeneous mixture of metal and metal oxide powders that produces an exothermic oxidation-reduction reaction. CS significantly increased the field of use for thermite systems [87]. An important type of CS is the reduction combustion synthesis. An example of this type of CS is:

$$
\mathrm{B}_{2} \mathrm{O}_{3}{ }^{(\mathrm{s})}+3 \mathrm{Mg}^{(\mathrm{s})}+\mathrm{Ti}^{(\mathrm{s})}=3 \mathrm{MgO}^{(\mathrm{l})}+\mathrm{TiB}_{2}{ }^{(\mathrm{s}, \mathrm{l})}+700 \mathrm{~kJ} / \mathrm{mol}
$$

where $\mathrm{TiB}_{2}$ is the desired product and $\mathrm{MgO}$ can be removed by leaching in acid solutions.

The reduction-CS method with additives, which is known as alkali metal molten salt assisted combustion, is widely used for the production of nano-powders, including metals ( $\mathrm{Ti}$, $\mathrm{Ta}, \mathrm{W}$ ) and metal carbides ( $\mathrm{TiC}, \mathrm{TaC}, \mathrm{WC}$ ), and was described in detail in a previous review [9]. In this process, the reducing metal, (e.g. $\mathrm{Mg}$ ) reacts with a transition metal oxide $\left(\mathrm{MeO}_{\mathrm{x}}\right)$ in the melt of alkali salt (e.g. $\mathrm{NaCl}$ ) to form nanosized reduced metal particles $(\mathrm{Me})$. Due to the heat generated by combustion reaction, salt melts at $\sim 1083 \mathrm{~K}$ and further nucleation of metal particles occurs in the molten $\mathrm{NaCl}$, which protects them from agglomeration and growth. The byproduct phases, i.e. $\mathrm{NaCl}$ and $\mathrm{MgO}$, can easily be leached out by washing the powder in an acid solution.

In recent years, due to the revolution in nanotechnology, there is a renewed interest in thermite systems, wherein both mixed metal oxide and metal powders are of nano-scale size, and 
hence the process is termed nano-thermite. The advantages of these systems, as compared to the previously investigated micron-scale reactive mixtures, are their extremely high ignition sensitivity, both by thermal and mechanical means, and record high reaction rates. The combustion aspects have already been discussed in detail in recent reviews [5, 18]. In this work we focus primarily on the microstructural aspects of nano-thermites and kinetics of their interaction, which are critical for application of such system for synthesis of nano-materials.

\subsection{Examples of nanothermite systems}

Though a vast range of nanopowders are available (aluminum, boron, carbon, silicon, nickel, and many oxides), a review of published literature ([12,25] and references in these reviews) shows that the main research objectives were thermite nanosystems with nano-Al (nAl) being used as a reducing element. Thus it is important to account for the morphological and microstructural aspects of such nano particles. The aluminum particle always has a thin layer of alumina on its surface. The $\mathrm{nAl}$ is typically stored under nitrogen $\left(99.9\right.$ vol. \% $\mathrm{N}_{2}$ with $50 \mathrm{ppm}$ $\mathrm{O}_{2}$ ) atmosphere in a glove box to prevent further oxidation and contamination. TEM images of Al nanoparticles (nAl) with different exposure time in air (0 to $90 \mathrm{~min}$ ) are shown in Fig. 13 (ad) [87]. It can be seen that the layer thickness is about $1 \mathrm{~nm}$ before exposure in air and reached an asymptotic value of about $4 \mathrm{~nm}$ after $30 \mathrm{~min}$.

Bismuth trioxide $\left(\mathrm{Bi}_{2} \mathrm{O}_{3}\right)$ nanoparticles were prepared by solution combustion synthesis using bismuth nitrate and glycine [88]. The microstructure of the reaction mixture, which contains the as-synthesized $\mathrm{Bi}_{2} \mathrm{O}_{3}$ nano-particles and fresh $\mathrm{nAl}$ powders with an average particle size of $100 \mathrm{~nm}$ is presented in Fig.13e. It was shown that such a system can burn with a glowing front propagation velocity equal to $2500 \mathrm{~m} / \mathrm{s}$ with an extremely rapid ( 10 $\mu \mathrm{s})$ gas pressure rise in the reactor. It was demonstrated [87] that the thickness of the $\mathrm{Al}_{2} \mathrm{O}_{3}$ layer significantly influences the reaction kinetics and thus the microstructural characteristics of the metal particles should be accounted when $\mathrm{nAl}$ is used for the synthesis of nanomaterials.

The next issue that one has to take into account is the amount of active metal in nAl particles, which depends on the size of the powder and methods of its production. Two nano aluminum powders manufactured by Argonide Corp. and Novacentrix are widely used by researchers. Argonide aluminum is produced by an exploding wire process, and is commonly referred to as ALEX aluminum. Novacentrix aluminum is manufactured using the inert gas 
condensation method following plasma heating. The properties of these powders are shown in Table 1.

The Argonide aluminum is found to consist of many fused spheres of nano aluminum mixed with some larger even micron-scale particles, while Novacentrix nAl consists of spherical particles with narrow particle size distribution. It was demonstrated that such characteristics lead to different optimum ratios between $\mathrm{Al}$ and the oxidizer, from the standpoint of degree of conversion, and thus results in different reactions rates [89]. Thus, parameters such as amount of active metal inside the particle, which is typically not recognized in micron-scaled systems, becomes an important issue in the case of combustion synthesis of materials in the reactive nanomixtures.

The studies with the nano-thermite systems also lead to the development of novel approaches for the preparation of reactive mixtures [90]. A wide variety of methods, like conventional mechanical and ultrasonic mixing, high energy ball milling (HEBM) and the solgel approach, have been used to achieve uniform mixing of nano-powders with a high contact surface area between the reagents. However, each of these methods exhibits some disadvantages. For example, physical methods, such as ultrasonic mixing and HEBM produce nano-mixtures with a random distribution of the oxidizer and fuel particles on the nanometer scale. The sol-gel approach provides better control over the reagents dispersion of the solid, however, typically results in the presence of undesired phases, e.g. $\mathrm{Al}_{2} \mathrm{O}_{3}$ (from dissolved $\mathrm{AlCl}_{3}$ salt) or $\mathrm{SiO}_{2}$ (from added silicon alkoxide). To overcome these limitations, a surfactant self-assembly method to produce nanostructured reactive mixtures (e.g. Al metal fuel and $\mathrm{Fe}_{2} \mathrm{O}_{3}$ oxidizer) with molecularlevel mixing has been developed [91]. This process involves several steps: (i) coating of $\mathrm{Fe}_{2} \mathrm{O}_{3}$ nanotubes with a surfactant, e.g. polyvinylpyrrolidone (PVP) by ultrasonicating in 2-propanol for $4 \mathrm{~h}$; (ii) removal of the residual surfactant by repeated cycles of centrifugation; (iii) drying of the extracted powder at $340 \mathrm{~K}$ for $12 \mathrm{~h}$; (iv) ultrasonication of the PVP coated nanotubes with nAl; (v) drying of the obtained mixture at $340 \mathrm{~K}$ for $12 \mathrm{~h}$ prior to the characterization. The morphology and microstructure of the as-prepared mixtures obtained by two different techniques i.e. self-assembly and conventional physical mixing are shown in Figure 14. The self-assembled $\mathrm{Al}-\mathrm{Fe}_{2} \mathrm{O}_{3}$ system (Fig. 14 a,b) clearly shows that high interfacial contacts between the $\mathrm{Fe}_{2} \mathrm{O}_{3}$ nanotubes and $\mathrm{nAl}$ particles have been achieved. Distinct segregated agglomeration of $\mathrm{Al}$ clusters was observed throughout the sample prepared by physical mixing (Fig 14 b, c). 
For example, it was demonstrated [92] that the $\mathrm{Al}+\mathrm{Fe}_{2} \mathrm{O}_{3}$ nano-thermite system prepared by the self-assembly approach exhibits significant enhancement in reaction kinetics as compared to nano-scale thermite samples prepared by simple physical solvent mixing. By increasing the intimacy between the fuel and oxidizer by self-assembly, solid-state diffusion between the reactants is significantly enhanced. It is also deduced that the interfacial contact area is more important than the reactant size, as revealed by the reduced thermal reactivity of physically mixed nano-sized reactants in contrast to those prepared by self-assembly. The latter conclusion is of vital importance for the future work on combustion synthesis of materials in reactive nanomixtures.

Another novel method for initial nano-mixture preparation was suggested in [93], with the main goal to reduce sensitivity of nano-thermite systems. Briefly, the metal oxide (e.g. $\mathrm{MnO}_{2}$ ) was enclosed inside a carbon nano-tube (see Figures 15a-c). The procedure involves impregnation of the CNTs with inner diameter $\sim 40 \mathrm{~nm}$ by the oxide containing precursor, i.e. melt of the manganese nitrate tetrahydrate $\mathrm{Mn}\left(\mathrm{NO}_{3}\right)_{2} \cdot \mathrm{H}_{2} \mathrm{O}$. After infiltration, the system was calcined to produce manganese oxide-particles encapsulated inside the CNTs (Fig.15c). This procedure was followed for the preparation of $\mathrm{MnO} / \mathrm{CNTs}+\mathrm{nAl}$ reactive mixture (Fig15.b) and reaction initiation. It was established that sensitivity of such a system was much lower as compared to conventional nanothermite mixtures (Fig.15a) and the combustion velocity was 10 times slower. This is a critical step towards using the nanothermite mixture for synthesis of materials, which requires precisely controlled conditions. The phase composition of the combustion product was also analyzed and found to contain phases such as graphite, akhtensite $\left(\mathrm{MnO}_{2}\right)$, ausmannite $\left(\mathrm{Mn}_{3} \mathrm{O}_{4}\right)$, manganese metal $(\gamma-\mathrm{Mn})$, corundum $\left(\mathrm{Al}_{2} \mathrm{O}_{3}\right)$, and aluminum carbide $\left(\mathrm{Al}_{4} \mathrm{C}_{3}\right)$. Based on additional analysis, the authors concluded that $\mathrm{MnO}_{2}$ was formed due to the metal $(\mathrm{Mn})$ re-oxidation after combustion process, while ausmannite $\left(\mathrm{Mn}_{3} \mathrm{O}_{4}\right)$, was formed from un-reacted $\mathrm{MnO}_{2}$, since some $\mathrm{Al}$ reacted with carbon to form carbide $\left(\mathrm{Al}_{4} \mathrm{C}_{3}\right)$.

\subsection{Some aspects of nanothermite systems}

The results of differential thermal analysis (DTA) shows that the onset reaction temperature in nano-thermite systems is much lower than micron size mixtures. For example in the $\mathrm{MoO}_{3}-\mathrm{Al}$ system, the main exothermic peak appears at a temperature of 900 and $400 \mathrm{~K}$ respectively when the $\mathrm{Al}$ particle size was $\sim 5 \mu \mathrm{m}$ and $\sim 50 \mathrm{~nm}$ respectively [94]. Based on the literature overview, 
one can make even more generalized conclusion [12] that in thermite $\mathrm{Me}_{\mathrm{I}}+\mathrm{Me}_{\mathrm{II}} \mathrm{O}$ systems, the micron-sized mixtures exhibit intense interaction at temperatures $\geq$ the melting point of reducing $\mathrm{Me}_{\mathrm{I}}$ (i.e. $\mathrm{Al}, \mathrm{Mg}$ etc), whereas nano-mixtures are extremely active at temperatures below melting point of any reagents. This important result suggests that for microstructural mixtures primarily liquid-solid or liquid-liquid reactions are responsible for the initiation of self-sustained combustion process, while in nano systems solely solid-state reactions may lead the process. Studying the kinetics of solid-phase reactions in nano-systems is a new field of science.

The range of the burning rates in such systems is even more remarkable. Different authors [87-93] report combustion wave velocities $\left(U_{c}>1000 \mathrm{~m} / \mathrm{sec}\right)$ at the level of the speed of sound in the reactive medium, emphasizing the absence of detonation. In our opinion, these data require both experimental validation and, possibly, revision of the interpretation of the results obtained. Indeed, all the previously reported data on the combustion velocities in micron-scale thermite systems (cf. [1]) are in the range 1-10 cm/s. None of the known kinetics models [94] can predict such a large increase in $U_{c}$ by just taking into account the size effect of the precursors. In addition, the maximum observed values for combustion velocities in nano-foils (see Section 5 of this review) do not exceed $100 \mathrm{~m} / \mathrm{s}$. It is worth noting that in the nanofoils, the sizes $(10-50 \mathrm{~nm})$ of the reagents even smaller than those for typical nano-thermite systems and their thermal conductivities are much higher as compared to the thermal conductivity of the porous mixtures of nano-powders. Based on all the above discussions, it can be concluded that the measured values of the hot spot propagation in the nano-thermite system are not related to the classical definition of the combustion velocity, but is the result of the combined effect of high temperature, gasification and gas flow in the constrained environments (capillary, channels and etc). In any case, the combustion of nanosystems requires new methods for measuring combustion parameters in heterogeneous systems with time scales in the range $\sim 1-10 \mu$ sec and heterogeneity scales below $0.1 \mu \mathrm{m}$.

\subsection{Reaction and microstructure transformation mechanisms of nanothermite system}

The specifics of combustion parameters such as burning rate, onset reaction temperatures, pressure evolution etc. were discussed in recent reviews [12, 25]. From a materials science standpoint, it is critical to understand the reaction and microstructure transformation mechanisms, which can be responsible for such extremely high rates of interactions in nano- 
thermite systems and hence in this part of the review, the focus is on this issue. Literature analysis suggests that there are currently three main mechanisms that are used to describe and interpret the reaction of nano- $\mathrm{Al}$ particles in an oxidizing atmosphere, including nano-thermites. In all of them, the layer of alumina on the surface of the $\mathrm{Al}$ metal particle plays a key role. It is worth noting that basic physical ideas for nano-Al oxidation are close to those formulated for the micron size metal particles [95, 96], where different mechanisms for the internal strains in the metal particle cladded by oxide layer have been discussed. The strain may lead to the rupture of the oxide layer, leading to the direct contact between metal and the oxidizer and thus accelerating the reaction. However, these ideas were reformulated for the conditions of nano-scale dimensions and high heating rates. The main features of these models are described below:

First is the so-called, diffusive oxidation mechanism under the internal pressure gradient, which was first justified experimentally [97] and then a model was developed [98]. The bottom line is that at high heating rates $\sim 10^{3} \mathrm{~K} / \mathrm{s}$ and temperatures above the m.p. of $\mathrm{Al}\left(660^{\circ} \mathrm{C}\right)$ large pressure gradients are present in the oxide shell of the nano-particles (Figure 16). If the coating thickness is less than $1 \mathrm{~nm}$ then the maximum of negative pressure is in the middle of the shell (Figure 16a), but if the oxide thickness has grown significantly (thickness $\gg>1 \mathrm{~nm}$ ), then a relatively wide region exists along the oxide shell (Figure 16b) with a constant large negative pressure ( 1-2 GPa). A natural effect of this pressure gradient will be the motion of atoms in the direction of decreasing pressure, which occurs in addition to the diffusive transport within the oxide shell. Thus, the pressure effect can be thought of as an effective convective flux of oxygen and aluminium ions in addition to the diffusive flux due to concentration. Oxygen will diffuse from the outer surface of the particle to the reaction surface gradient (see Figure 16b), while aluminium will diffuse from the metal/metal oxide interface to the reaction surface, leading to the formation of hollow alumina particles.

It was found that under the suggested model the kinetics law can be presented in the following form:

$$
\mathrm{t} \propto \mathrm{r}^{1.6 \pm 0.1}
$$

where $t$ is the time required for a particle to react to a degree of conversion (e.g. 50\%) and $r$ is the initial radius of the particle. If this process was just a surface reaction in the free molecular regime, one should expect a linear relationship. But here, the diffusion of species through the aluminium oxide shell is the rate limiting step and hence it is not a purely surface process. If we 

pressure gradients, then the power law obtained is $\mathrm{t} \propto \mathrm{r}^{2}$ and is in agreement with the shrinking core model in the absence of pressure gradients. It is noted that this rapid diffusion process may be enhanced by the rupture and thinning of the oxide shell.

Second is, the melt-dispersion mechanism (Figure 17), which, as it is stated, works at temperature above the melting point of $\mathrm{Al}$ and very high heating rates $>10^{6} \mathrm{~K} / \mathrm{s}[99,100]$. Briefly, it was shown that at fast heating rates, the alumina shell of nanoparticles does not break until $\mathrm{Al}$ melts. The main geometric parameter that determines stresses in the Al core-shell system is the ratio of $\mathrm{Al}$ core radius $\mathrm{R}$ to oxide thickness $\delta, M=\mathrm{R} / \delta$. It was found [99-101] that for $M<19$, the entire $\mathrm{Al}$ particle melts before the oxide shell fractures. Melting of $\mathrm{Al}$ is accompanied by a $6 \%$ volume expansion which creates high dynamic pressures (1 to $3 \mathrm{GPa}$ ) in the liquid core. Pressure of such magnitudes overloads the alumina shell with the hoop stresses $\sigma_{\mathrm{h}}$ that exceeds the ultimate strength of alumina $\sigma_{u}$ and causes the shell's dynamic fracture and spallation. After oxide spallation, the pressure within the liquid $\mathrm{Al}$ remains unchanged while at the bare $\mathrm{Al}$ surface the pressure is of the order of $10 \mathrm{MPa}$ only due to surrounding gas pressure and surface tension. Then an unloading wave propagates from the surface to the center of the particle and creates a tensile pressure at the particle center of 3 to $8 \mathrm{GPa}$. Such a pressure exceeds the strength (cavitation limit) of molten $\mathrm{Al}$, and disperses the liquid $\mathrm{Al}$ into small bare clusters that fly at a high velocity $(100-250 \mathrm{~m} / \mathrm{s})$. Oxidation of these clusters is not limited by diffusion through the initial oxide shell.

The third mechanism is also a diffusion-type oxidation model, but accounts that the phase transformations in the alumina shell and was established based on the experimental results obtained by conventional thermo-gravimetric analysis (TGA) [102, 103]. Figure 18 shows a characteristic sequence of changes in the alumina scale growing on the particle surface. It was suggested that the entire oxidation process can be divided into four stages and specific processes occurring during each stage are illustrated schematically. The natural amorphous alumina layer covering the particle initially grows slowly during the low temperature oxidation stage I. The energy of the oxide-metal interface stabilizes the amorphous oxide only up to a critical thickness of about $5 \mathrm{~nm}$. When the critical thickness is approached or when the temperature becomes sufficiently high, the amorphous oxide transforms into $\gamma$-alumina. The density of $\gamma$-alumina exceeds that of amorphous alumina, and the smallest $\gamma$-alumina crystallites have a size of about 
$5 \mathrm{~nm}$. Thus, if prior to the phase change, the thickness of the amorphous layer was less than 5 $\mathrm{nm}$, the newly formed $\gamma-\mathrm{Al}_{2} \mathrm{O}_{3}$ crystallites no longer form a continuous layer covering the aluminium surface. As a result, the rate of oxidation increases rapidly at the beginning of stage II as shown in figure 18. As the openings in the oxide coating heal, the rate of oxidation decreases. Eventually, a regular polycrystalline layer of $\gamma-\mathrm{Al}_{2} \mathrm{O}_{3}$ forms by the end of stage II. The growth of $\gamma-\mathrm{Al}_{2} \mathrm{O}_{3}$ continues in stage III for which the oxidation rate is limited by the inward grain boundary diffusion of oxygen anions. Stage III ends when the high temperature destabilizes the transition of alumina polymorphs. The stable and denser $\alpha$-alumina polymorph starts forming by the end of stage III; stage IV is considered to start when the oxide scale is completely transformed to $\alpha$-alumina. When first individual $\alpha-\mathrm{Al}_{2} \mathrm{O}_{3}$ crystallites begin to form by the end of stage III, the thickness of the $\gamma-\mathrm{Al}_{2} \mathrm{O}_{3}$ layer decreases, and the oxidation rate abruptly increases. Once most of the oxide layer is transformed to coarse and dense $\alpha-\mathrm{Al}_{2} \mathrm{O}_{3}$ crystallites resulting in continuous polycrystalline coverage, grain boundary diffusion slows down, and the oxidation rate decreases rapidly.

At high heating rates the thickness of the $\gamma$-oxide layer may be smaller than some critical value $\left(\mathrm{h}_{\mathrm{m}}\right)$, which leads to the formation of a discontinuous oxide layer with no diffusion resistance. In this case, the rate of diffusion was controlled by the parent oxide layer, i.e. amorphous or $\gamma$-oxide, for stages II and III respectively. However, for practically interesting, high heating rates, the parent oxide may disappear completely even before the layer of the new polycrystalline phase becomes continuous, that is, before the limiting thickness $h_{m}$ is reached and a portion of the aluminium surface becomes directly exposed to the oxidizing gas environment.

All of the above models, which have some similarity, but in general lead to different kinetics laws, were used to describe extremely rapid burning rates in the nano-thermite systems. The question is: which of them describes the real nature of the reduction type combustion in nano-scale heterogeneous media? In our opinion each of these mechanisms has some strong points as well as weaknesses.

Indeed, cracking of the $\mathrm{Al}_{2} \mathrm{O}_{3}$ layer, which is the conceptual idea of the second model and possibility of which is mentioned in the first one, looks reasonable, for example, it explains the rapid reaction in the system where one of the nano-precursor ( $\mathrm{Al}$ ) has very thick layer of oxide. But all the models suggested that rupture mechanisms can work at temperature above Al melting point $(912 \mathrm{~K})$. In this case how can one explain the values of the ignition temperature for such 

exposed to the oxidizing atmosphere, what will be the rate of chemical interaction? It was reported that self-ignition temperature for oxygen free titanium particles in air equals to room temperature and combustion occurs with very high velocity [104].

Also, we suppose that it is vital to include the phase transformation aspects to the combustion mechanism, as it was suggested in the third model. However, for the extremely high heating rates in the combustion front and very short reaction time, the question arises regarding the kinetics of these phase transformations. Is it enough time in the reaction front to follow all these equilibrium routes? Finally, we have strong beliefs that without use of extremely high (experimentally not proven) diffusion coefficients none of the models, which are based on the diffusion through the product layers, can explain the low values of observed onset reaction temperatures and high rates of combustion wave propagation. We have discussed these issues in our recent review [12] and concluded that more in-situ experiments are required in order to define with enough confidence the characteristic reaction time (rates) for nano-thermite reactions.

From the standpoint of materials synthesis several issues should be outlined. First let us note that all these reaction models involve microstructural aspects, which are connected to the morphological or/and phase transformations. Moreover these microstructural transformations play a key role in the suggested mechanisms and allow one to explain the extremely fast observed interaction rates. Second these chemicals models are strongly connected to the mechanical behavior of the heterogeneous system, i.e. different types of internal stresses and strains, which lead to the morphological transformations. The above features are very closely correlated to the concept of, so-called, structural macrokinetics (SM) [2, 94]. The SM deals with the processes of structural (crystal lattice, elemental composition, morphology, etc.) transformations, which take place under the conditions of intensive heat and mass transport. The main objective of SM is to control not only the reaction/combustion rate, but also more importantly to govern the microstructure of the reactive media to produce materials with desired properties. There is beyond doubt that very soon combustion in the mixtures of nano-powders will not only be used for rapid release of heat and pressure increase, but also for synthesis of 
unique materials. And such structure related models are a good fundamental base for the development of this new class of nano-structured materials.

\subsection{Applications of nanothermite systems}

Thermites are also used for cutting or welding steel such as rail tracks (Goldschmidt reaction), without requiring complex or heavy equipment [105]. It is not clear if $\mathrm{nAl}$ or $\mathrm{nMg}$ will find applications for the purification of ores, because of the cost and safety issues. However, such nano-reactive systems can be used as cutting tools, especially in some harsh environments (salt water etc.) [106]. The nanothermites, with their fast reaction velocities, have been also proposed for use as igniters and lead-free primers for ammunition [107]. This example shows that the use of energetic nanomaterials may allow the replacement of toxic substances - lead and mercury salts as primary explosives, while being less expensive than other replacements, such as silver azide or fulminate. Also, certain applications of solid-propellant micro-thrusters, such as course correction of high-velocity projectiles or microsatellites, require that the actuation time be as short as possible. It was shown that nanothermite composites are capable of producing reaction propagation rates similar to those of conventional primary explosives, but with pressure levels well below that of solid explosives. This characteristic makes composites a promising candidate for fast-impulse microthruster applications [108]. It was found that nanothermite composites are capable of producing shock waves with Mach numbers up to 2.44 in an air-filled tube [109]. In general, micro- and nano-electromechanical systems and sensors are extremely promising fields for nanothermites [110]. In addition, the shock wave if produced by reaction of super-thermites can be used for production of nanomaterials such as nanodiamonds [111].

Another specific application is related to gas generators [112]. It was demonstrated that among a variety of $\mathrm{MeO}-\mathrm{Al}$ nanothermite systems $(\mathrm{Me}=\mathrm{Fe}, \mathrm{Mo}, \mathrm{W}, \mathrm{Mn}, \mathrm{Cu}, \mathrm{B}) \mathrm{Bi}_{2} \mathrm{O}_{3}+\mathrm{Al}$ composition, where metal oxide nanopowder was produced by solution combustion synthesis method, shows the highest pressure peak ( 10MPa) with $\Delta \mathrm{P} / \Delta \mathrm{t}$ of $500 \mathrm{GPa} / \mathrm{s}$. The maximum P$\mathrm{V}$ value obtained using a $0.1 \mathrm{~g}$ sample with as-synthesized well crystalline $\mathrm{Bi}_{2} \mathrm{O}_{3}$ and nano aluminum mixture was $707 \mathrm{~Pa} \mathrm{~m}^{3}$. This is much higher than that reported in the literature $33 \mathrm{~Pa}$ $\mathrm{m}^{3}$ for the same sample mass.

Thus all of the above mentioned applications are mostly related to such properties of nano-thermites like extreme rapid reaction rates and high gas evolution. The question is whether these systems can be also used for materials synthesis. Based on our experience in the field of CS 
we can make a prognosis that soon this research work will appear where the reactive mixtures of nano-powders will be used for the synthesis of unique nano-composites, cermets and alloys. The near future will validate this prediction.

\section{Multilayer reactive nano-foils (MRNF)}

The basic method for the production of reactive nano-foils is the layer-by-layer magnetron deposition, in which the cold substrate, placed on the rotating holder, passes by turns through spatially separated flows of atoms, sputtered from different magnetron targets. Details of the method are described elsewhere $[11,12,113]$. Relatively flat continuous layers of the sputtered elements (usually metals) deposit alternatively one over another. Thickness of the layer may vary from several nanometers to microns, while number of the layers "stacked" in one foil may achieve several thousands. The overall thickness of the foil is limited by mechanical stresses accumulating in the heterogeneous lamellar structure composed of dissimilar materials, and usually do not exceed 200 micron. The typical microstructure of the magnetron deposited multilayer reactive nano-foil is shown in Figure 19 a,b. Although the Ni/Al MRNF's are the most studied system of numerous experimental and theoretical works, many other Me/Al bimetal nano-foils, where $\mathrm{Me}=\mathrm{Ti}, \mathrm{Co}, \mathrm{Pd}, \mathrm{Pt}, \mathrm{Cu}, \mathrm{Nb}$, Ta, were obtained using this method [12]. Besides, some metal/nonmetal MRNF's have been produced recently by magnetron sputtering, including $\mathrm{Nb} / \mathrm{Si}[114]$ and $\mathrm{Ti} / 2 \mathrm{~B}[115]$.

General limitations of the magnetron sputtering are the high cost and relatively low yield. The quest for alternative, efficient production methods led to the development of the multiple cold rolling technique [116-121], also named as accumulative roll bonding process [121,122]. A bimetal stack of alternate foils undergoes cold rolling several times, which results in reduction of the initial thickness by an order of magnitude or even more. For example, using a stack of original $\mathrm{Ni}(18 \mu \mathrm{m}$ thick) and pure $\mathrm{Al}(25 \mu \mathrm{m})$ foils, the multilayer foil with bilayer thickness of $5 \mu \mathrm{m}$, total thickness of $0.85-0.9 \mathrm{~mm}$ was obtained [117]. Modification of this method involves hot rolling of the bundle of metal sheets (foils) in vacuum at $1000-1300 \mathrm{~K}$ as a first technological stage, which provides formation of multilayer bulk material [123]. Instead of hot rolling, hot vacuum pressing can be applied at the first stage for the same purpose of producing bulk multilayer rolling billet [124]. The second stage is multiple cold rolling of this material in air. The schematic representation of this method is shown in Figure.20a. It was revealed that the 

However, the real nano-laminates were obtained in the systems of nonreactive, immiscible metals. Thus, multilayer foil with the flat continuous nano-layers and advanced mechanical properties was obtained in the $\mathrm{Cu}-\mathrm{Nb}$ system: bilayer thickness was about $10-20 \mathrm{~nm}$ [125]. Nanolaminate composites with tortuous, nonuniform layers were reported for the $\mathrm{Cu}-\mathrm{Ag}$ system with limited solubility [126]. For the reactive systems, with the ability of the intermetallic phases formation, the thickness of individual metal layers in the laminate shoots up to several microns: the microstructures presented in the literature possess bilayer periodicity over $50 \mu \mathrm{m}$ for the $\mathrm{Cu}$ Ti [124], about $20-30 \mu \mathrm{m}$ for the Ti-Ni [127], and $140 \mu \mathrm{m}$ for the Ni-Al [128] composites. Therefore, mechanical methods allow production of nanometer-scale laminates in the nonreactive immiscible systems, and micrometer-scale laminate composites of the reactive metals.

Non-uniformity, intermittence and tortuosity of the layers are the most common microstructure defects of the reactive multilayer foils produced by mechanical methods. Microstructure of the cold-rolled Ni/Al film presented in Figure 19 c,d shows that the thickness of individual layers varies in the range $0.1-10 \mu \mathrm{m}$. These effects are probably caused by difference in mechanical properties of $\mathrm{Ni}$ and $\mathrm{Al}$ (yield strength, hardness, etc.) and sliding of the layers relative to each other. In order to improve contacts between layers, careful cleaning of the surfaces of initial foils is used shortly before the cold or hot rolling, e.g., wire-brushing and cleaning with acetone for the cold rolling [119], grinding with abrasive paper for the hot+cold rolling [124], etc. The pre-treatment of the initial metal foils or sheets removes surface oxides and other impurities, and creates proper surface geometry for better adhesion of the layers.

The third branch of production methods, which should be noted here, is a combination of high-energy ball mill and cold rolling and schematically shown in Figure.20 b. The idea of this combination, suggested in [4], has been accomplished as a technological method in [129]. Figures 19 e, f show microstructure of the Ni/Al sub-micrometer laminate ribbon produced by cold rolling of the high-energy ball milled composite powders (see Section 2). Evidently, the nanometer-scale $\mathrm{Ni}$ and $\mathrm{Al}$ layers are present in the material. The ball milled and cold rolled foils possess relatively high non-uniformity, intermittence and tortuosity, as compared to the 


\subsection{Kinetics of multilayer reactive nano-foils}

Kinetics of the heterogeneous reactions strongly depends on the temperature - time regime of the process. Most of the systems under consideration react at growing temperature conditions, when the temperature rises due to external heating or internal reaction self-heating. All possible reaction stages and intermediate products can be revealed at slow heating, e.g., using the differential scanning calorimetry (DSC) method, where the testing samples are heated at the rate of $0.1-1 \mathrm{~K} / \mathrm{s}$. The reaction route changes dramatically, when the heating rates increases up to $10^{5}-10^{7} \mathrm{~K} / \mathrm{s}$, which are typical parameters of the combustion wave front. When the heating rate is very high, most of the reaction stages and products do not occur; the reaction mechanism is simplified and often reduced to one high-temperature stage. For instance, in the Ni/Al nanolaminates, slow DSC heating revealed the formation of several intermediate phases, including primary phase $\mathrm{NiAl}_{3}$, metastable $\mathrm{Ni}_{2} \mathrm{Al}_{9}$, and dominant growing $\mathrm{Ni}_{2} \mathrm{Al}_{3}$, preceding the appearance of the final B2 $\mathrm{NiAl}$ phase [130,131]. Direct measurements of the phase transformations during combustion of the Ni/Al multilayer nanofoils, made by time-resolved Xray micro diffraction using synchrotron radiation, have shown that the reaction occurred in one stage, and NiAl was the only product [132]. Probably, this rule is applicable also for other compositions of the MRNF. A nanocalorimetry method, specially developed for investigation of heterogeneous reaction mechanisms in the nano-layer systems at high heating rates, was applied, in conjunction with modeling, to the Ni/Al foils with $50 \mathrm{~nm}$ and $60 \mathrm{~nm}$ bilayers [133]. The results have shown one exothermic reaction stage in the temperature range $600-1000 \mathrm{~K}$, with the maximum chemical self-heating rate about $4 \times 10^{5} \mathrm{~K} / \mathrm{s}$.

Two intriguing features of the MRNFs are the very low temperature of the reaction initiation and high velocity of the reaction wave propagation (gasless combustion). Compilations of the experimental data were presented in previous reviews [11, 12]. Self-sustained reaction can be initiated at temperature of $425-474 \mathrm{~K}$, much below the melting point of any metal component, and $570-770 \mathrm{~K}$ lower than the ignition temperature of micro-heterogeneous powder mixtures with similar compositions. Commonly, solid state diffusivity is negligible at that temperature, however, nano-heterogeneous materials may possess different transport properties. 
Thus, nanocalorimetry measurements [133] gave the following expression for calculating inter diffusivity in the $\mathrm{Ni} / \mathrm{Al}$ nanolayers at temperature $\mathrm{T}=720-860 \mathrm{~K}$ :

$$
\mathrm{D}=\left(2.79 \times 10^{-10}\right) \exp \left(-\frac{6900}{\mathrm{~T}}\right)
$$

Calculation by this formula gives $\mathrm{D}=(2.0 \div 9.7) \times 10^{-14} \mathrm{~m}^{2} / \mathrm{s}$, which is orders of value higher than expected from the reference data on bulk materials for the corresponding temperature range. Molecular dynamics simulation of the process of reaction initiation (self-ignition) in the $\mathrm{Ni} / \mathrm{Al}$ nanolaminates demonstrated a significant role of interphase boundary misfits in the reaction initiation [134]. Increasing of the misfit stress up to $6.87 \%$ results in decrease of the calculated reaction ignition temperature from $800 \mathrm{~K}$ to $450 \mathrm{~K}$. Another hypothesis on the causes of hyperreactivity is that thin intermixed layers on the interphase boundary, which form during deposition can be observed by TEM (see fig.19,b), and serve as precursors of the reaction, lowering activation barrier for the reaction initiation. As discussed in Section 2, similar layers possessing lower self-ignition temperature can also be observed in the high-energy ball-milled mixtures (fig.19 e). Thus, initiation of the self-sustained reaction arises from solid state processes, which occur with unusually high rates at relatively low temperature due to enhanced diffusivity in the nanostructure heterogeneous materials and, possibly, due to nanoscale solid precursors. The mechanisms of these solid state processes are the subjects for the nearest future investigation.

Once initiated, the exothermic reaction releases heat sufficient for a sharp increase of the temperature above the melting point of at least one reactant. Hence, the main stage of reaction includes interaction between solid refractory metal layers and molten compounds. The solidliquid reaction mechanism must explain the above mentioned high velocities of the reaction wave propagation, which can reach $80-90 \mathrm{~m} / \mathrm{s}[11,12]$. One of the existing models presumes that a continuous layer of solid product forms at the interphase boundary, and the reaction proceeds due to diffusion of the reactants through this layer, as shown schematically in figure 20 a. According to this model, in the $\mathrm{Ni} / \mathrm{Al}$ reactive multilayer foils, $\mathrm{Ni}$ and $\mathrm{Al}$ atoms have to diffuse through the NiAl solid layer. Evaluation of the diffusivity obtained as best fits of the experimentally measured reaction wave propagation velocity [135] gave:

$$
\mathrm{D}=\mathrm{D}_{0} \exp \left(-\frac{\mathrm{E}}{\mathrm{RT}}\right)=\left(2.18 \times 10^{-6}\right) \exp \left(-\frac{16478}{\mathrm{~T}}\right)
$$

where $\mathrm{R}=8.314 \mathrm{~J} / \mathrm{mol} \cdot \mathrm{K}$-is the universal gas constant, $\mathrm{E}=137 \mathrm{~kJ} / \mathrm{mol}$ is the activation energy. Despite of the strong differences in the pre-exponent factors and activation energies, calculations 

region. At $\mathrm{T}=860 \mathrm{~K}$ equation (6) gives $\mathrm{D}=1.2 \times 10^{-14} \mathrm{~m}^{2} / \mathrm{s}$, which is about 8 times lower than the result of calculation by equation (5), and still higher than expected from data on bulk materials.

Calculations by using (6) for the high-temperature range led to the following values: for the adiabatic combustion temperature $(1900 \mathrm{~K}) \mathrm{D}=4.0 \times 10^{-10} \mathrm{~m}^{2} / \mathrm{s}$; for the melting point of $\mathrm{Ni}$ $(1728 \mathrm{~K}) \mathrm{D}=1.7 \times 10^{-10} \mathrm{~m}^{2} / \mathrm{s}$; for the eutectic point $(1658 \mathrm{~K}) \mathrm{D}=1.1 \times 10^{-10} \mathrm{~m}^{2} / \mathrm{s}$. These values are much higher than the diffusivities of the $\mathrm{Ni}$ and $\mathrm{Al}$ in the $\mathrm{B}-2 \mathrm{NiAl}$ determined from the independent measurements [136], which can be described for the stoichiometric (50/50 at\%) nickel aluminide as

$$
\mathrm{D}=\left(3.45 \times 10^{-5}\right) \exp \left(-\frac{34881}{\mathrm{~T}}\right)
$$

Equation (7) was derived from the experimental data in the temperature range $1050-1630 \mathrm{~K}$. Extrapolating this equation for higher temperatures, one can estimate the diffusivity of $\mathrm{Ni}$ in $\mathrm{NiAl}$ at the combustion temperature as $4.1 \cdot 10^{-13} \mathrm{~m}^{2} / \mathrm{s}$, or at Ni melting point as $6.8 \times 10^{-14} \mathrm{~m}^{2} / \mathrm{s}$. According to molecular dynamic simulation, the diffusivity of $\mathrm{Al}$ in solid $\mathrm{NiAl}$, is about 2.5 times slower than that of $\mathrm{Ni}$ [137]. The results show that solid state diffusivity, determined from independent experiments and simulations, is $10^{3}-10^{4}$ times lower than that is required for the explanation of high gasless combustion velocity within the frames of the model shown in figure 20 a. One can debate, of course, that diffusivity in nano - materials is higher due to large concentration of crystal lattice defects, grain boundaries and other specific features of the nano state of the matter. However, in the considered systems experimental proofs and measurement of the diffusivities in the highly-defective solids are not available yet. On the other hand, selfdiffusion of $\mathrm{Ni}$ in the Al-Ni melt, experimentally measured by means of quasielastic neutron scattering at $1928 \mathrm{~K}$, was found to be equal to $4.9 \cdot 10^{-9} \mathrm{~m}^{2} / \mathrm{s}$ [138]. Therefore, if we assume that the reaction is controlled by diffusion in the melt, extremely high rates of the reaction wave propagation find their obvious explanation.

The reaction model based on direct dissolution of the refractory compound into the melt and liquid-state diffusion is presented schematically in figure $20 \mathrm{~b}$. This model assumed that the grains of solid products do not form a permanent layer, and therefore, liquid state diffusion takes place in the gaps between the grains. This mechanism was experimentally found in the quenched $\mathrm{Ni} / \mathrm{Al}$ MRNF reaction waves [139]. Microstructure of the reaction zone of the gasless Ni/Al combustion wave, obtained by quenching of the reaction process, is shown in Figure 21a. It is 
evident that the solid product consists of separate spherical nanosized grains, which do not form a continuous solid layer. A similar conclusion can be made from the molecular dynamics simulations of the reaction, an example of the calculated atomic distribution is shown in figure $21 \mathrm{~b}$ [140]. Thus, the model of direct dissolution into the melt and precipitation of separate grains adequately describes the experimentally observed features of the heterogeneous hightemperature reactions in the nano-layer gasless energetic materials.

The reaction mechanisms in the multilayer foils produced by mechanical methods (cold rolling) are less puzzling. Since the thickness of the layers realized by this method so far lies in the micrometer-scale range (see fig.19 c,d), the combustion front propagation velocity does not exceed several centimeters per second [141]. Such values can be explained by solid or liquid state diffusion mechanisms and, probably both of these processes occur in the mechanically structured multilayers.

\subsection{Applications of the multilayer foils}

Applications of the MRNFs follow from their thermodynamic properties and reaction mechanisms. Due to the high reaction rate and fast heat release, these functional materials find applications in joining (bonding) materials, in the reaction initiating systems, for enhancement of the heat and blast effects of energetic materials, etc. For joining purposes, the reaction foil is placed, together with the solder layers, between two materials (items), similar or dissimilar, which are to be joined. The precise amount of heat, instantly released by the foil after reaction initiation, melts the solder layers and joins the materials, without a damaging effect on their structure and properties. A wide spectrum of different materials and items, from refractory metals and ceramics to sensitive microelectronic components (e.g., LED crystals) and amorphous metal glasses can be soldered. More detailed reviews of this class of applications can be found in $[11,113]$. Magnetron - deposited Ni/Al multilayer nanofoils are the most used, commercially available soldering composite, characterized by a heat release $\Delta \mathrm{H}=118 \mathrm{~kJ} / \mathrm{mol}$ and a selfheating adiabatic temperature $\mathrm{T}_{\mathrm{ad}}=1911 \mathrm{~K}$. Some recent examples of the low-temperature bonding in microsystem technology are: IR-emitter bonded on a covar socket; quartz strain gauge bonded on a steel membrane $[142,143]$. Higher (as compared to Ni/Al) heat release and temperature can be obtained in the $\mathrm{Pd} / \mathrm{Al}\left(\Delta \mathrm{H}=163 \mathrm{~kJ} / \mathrm{mol} ; \mathrm{T}_{\mathrm{ad} .}=2270 \mathrm{~K}\right)$ and $\mathrm{Pt} / \mathrm{Al}(\Delta \mathrm{H}=201$ $\mathrm{kJ} / \mathrm{mol} ; \mathrm{T}_{\mathrm{ad} .}=2580 \mathrm{~K}$ ) systems. Formation of the non-desirable intermetallic phases [144] and 
relatively high cost of the $\mathrm{Pt}$ and $\mathrm{Pd}$ hinder $\mathrm{R} \& \mathrm{D}$ of these compositions; however, successful applications have already been reported [145]. A specific feature of the work is that very thin $(0.9-4.5 \mu \mathrm{m})$ multilayer nanofilms (bilayer thickness $75-400 \mathrm{~nm}$ ) were deposited directly on the components of microelectronic devices. After deposition, the proper pattern of the reactive film on the substrate was created by wet etching. Successful bonding was obtained using $\mathrm{Pd} / \mathrm{Al}$ and $\mathrm{Ti} / \alpha-\mathrm{Si}$ multilayers.

The second branch of the practical problems, solved by utilization of the MRNFs, relates to the pyrotechnic and military applications, such as enhancing the blast effect of energetic materials or as elements of various initiators [146, 147]. Initiation and reaction of the multilayer nanofoils under fast dynamic compression have been studied in different experimental works using several methods of the dynamic loading. Shockless laser-driven compression of the Ni/Al MRNFs was obtained by means of the laser beam pulse with wavelength $351 \mathrm{~nm}, 3.7 \mathrm{~ns}$ duration and intensity $(3.8 \div 7.6) \times 10^{12} \mathrm{~W} / \mathrm{cm}^{2}[120]$. The laser beam was not focused at the foil surface, but at the intermediate ablator reservoir, located at short distance before the foil. Expanding dense plasma, generated due to laser exposure of the ablator-reservoir, compressed the foil without formation of the shock wave. Fully reacted NiAl with grain size $\sim 500 \mathrm{~nm}$ was found in the compressed region; however, the exothermic reaction did not self-sustain and did not propagate throughout the foil. Similar results were obtained for the direct laser shock-wave loading, when the foil surface was irradiated by the light beam with intensity $2.68 \times 10^{11}-$ $1.28 \times 10^{13} \mathrm{~W} / \mathrm{cm}^{2}$, and pulse duration 3 and $8 \mathrm{~ns}$ : the laser shock created craters, spallation, and extreme damage in the laminates, but did not generate a self-propagating reaction through the sample [117]. These results are puzzling, because the self-sustaining reaction in the Ni/Al nanofoils can be easily initiated by weak electric spark, or by local heating up to $473-573 \mathrm{~K}$. Probably, the reason is that the duration of the laser pulse is not enough to form the preheated region comparable with the thickness of preheating zone of the steady reaction wave, which is required for ignition. It is also possible that destruction and fragmentation of the exposed region prevents formation of the preheated zone, because the hot pieces of the foil fly out of this zone.

A different result was obtained using mechanical impact [148]. The flyers made of different materials (e.g., $\mathrm{Cu}$ or $\mathrm{Al}$ ) were accelerated by laser beam, up to velocity of $1.4 \mathrm{~km} / \mathrm{s}$, and struck the $\mathrm{Ni} / \mathrm{Al}$ vapor-deposited MRNF. It was found that the reaction initiation threshold impact velocity was $270 \mathrm{~m} / \mathrm{s}$ for foils with $30-\mathrm{nm}$ bilayer spacing, and that the reaction to the $\mathrm{NiAl}$ 

shock wave propagation inside the laminates, made using real microstructures of the cold rolled $\mathrm{Ni} / \mathrm{Al}$ materials, allows to conclude that the orientation of the layers can greatly affect the dissipation and dispersion of the shock waves [118].

The electrically exploding foil initiators, due to their safety and reliability, are widely used for military and civilian applications. Additives of the $\mathrm{Al} / \mathrm{CuO}$ magnetron sputtered MRNF to the exploding foil initiator was studied in the work [146]. It was concluded that the reactive multilayer foil has the potential to decrease the initiation threshold, which will lead to a smaller volume of initiator, further increasing safety and reliability. A simple micro-initiator device was suggested using Ni/Al nanolaminates [147]. The ignition power threshold was found as small as $3 \mathrm{~mW}$ with the ignition delay about $0.63 \mathrm{~s}$. Unlike the exploding initiator, the latter device produces only heat, without any mechanical impact, and, therefore, cannot be used for initiation of the explosives.

The gasless exothermic reaction in the MRNF, at some conditions, can initiate the solid - gas reaction of oxidation between unreacted components of the same film and gas environment (oxygen, etc.). This solid-gas reaction increases the energy output of the reactive material (foil) and duration of the heat release. The potential application of the reaction foil as "self-initiator" in oxidizing environments was demonstrated for the Ti/2B [115] as well as for the $3 \mathrm{Al} / 2 \mathrm{Ni}, 3 \mathrm{Al} / \mathrm{Zr}$, and $\mathrm{Al} / \mathrm{Zr}$ [149] compositions.

Finally, it should be noted that most of the considered multilayer reactive nano - foils can be used for the combustion synthesis of fine-grained (sometimes, nanocrystalline) intermetallics. However, taking into account the relatively high cost of the MRNF production, this method does not look like a promising and economically effective production route. Some of the multilayer foils possess interesting mechanical or physical properties, e.g., high tensile strength or superconductivity. Consideration of these properties is beyond the scope of this review. 


\section{Conclusions}

Four different types of heterogeneous combustion systems have been discussed. The first system consists of the composite particles prepared by HEBM in which solid precursors are intermixed on the nano-scale level. The second system involves an amorphous gel formed after drying of the reactive aqueous solution in which precursors are mixed on the molecular level. The third system is a mixture of the nano-sized powders. The last system is a micron-thickness foil that consists of alternative nano layers of metallic reactants. These systems have several common features: (i) they are reactive enough to allow propagation of the self-sustained combustion wave; (ii) such self-sustained reactions can be used for synthesis of useful products and (iii) the precursors are mixed on the nano-scale level.

The last feature distinguishing these nanostructured reactive systems from the conventional reactive mixtures is that they are used for materials synthesis via combustion based routes. The above analysis reveals that being very different by nature these systems possess some similar properties. First of all they have low onset reaction temperatures, which are typically below the melting points of the precursors. It is well recognized that for the micron-size reactive powder mixture the onset reaction temperature equals or above the melting point of the less refractory precursor (or system eutectic). This effect is primarily related to the extremely low contact surface area between the reactants in such mixtures. All considered nano-systems owing to the preparation methods and small dimensions of the reactants possess much higher contact surface area between reactants thus allowing reaction initiation solely due to solid state processes.

An even more striking match can be found between the combustion of nano-foils and HEBM composite particles. It can be seen that the inner microstructure of individual composite particles is similar to those for the nano-foils of the same composition, but is not as regular. Otherwise it looks like that ignition temperature and combustion characteristics of such composite particles are comparable with corresponding reactive nano-foils. Lower combustion velocity which is detected for the mixture of the HEBM-particles is due to the smaller thermal diffusivity of the powder media as compared to pore-free foil.

On the other hand, one can find pronounced differences in the combustion of these nano-systems. Indeed, the range for the experimentally measured values of the combustion 
velocity extends from $\sim 1 \mathrm{~mm} / \mathrm{s}$ for sol-gel combustion, through tens of $\mathrm{cm} / \mathrm{s}$ for HEBM powder mixture and tens of meters per seconds for nano-foils, to $10^{3} \mathrm{~m} / \mathrm{s}$ for nano-thermites. While we doubted the correctness of the later value, it is still an impressive range of values. Interestingly, the lowest combustion velocities are observed for the system where the precursors are mixed on the molecular level (sol-gels). This effect can be explained by relatively low exothermicity of the systems, i.e. maximum measured combustion temperatures are on the order of $1000 \mathrm{~K}$, as well as by large amount of gas-phase products, which take away heat from the reaction front $[150,151]$. It is worth noting that in sol-gel systems, the combustion front can propagate in a self-sustained manner even if the maximum combustion temperature is $\sim 500 \mathrm{~K}$. Before it was believed that such waves may propagate only if combustion temperature is on the order of $2000 \mathrm{~K}$ [152].

Finally, one or more similarities between all considered systems are outlined. It looks like all of them can be used to synthesize nanomaterials. In general, it is a challenging task to produce nano products by using high temperature heterogeneous combustion processes. It looks like the short term high temperature conditions of the combustion wave, as well as intensive gasification (sol-gel and nanothermites systems) and initial structures of the precursors (HEBM composite particles and nano-foils) facilitate the formation of nano-sized structures. However, the application aspects of reactive nano-systems require further investigation.

\section{Acknowledgements}

One of the authors, Dr. Aruna thanks Director, CSIR- NAL for the constant encouragement.

\section{References}

1. A.G. Merzhanov A.G, V.M. Shkiro, I.P. Borovinskaya (Institute of Chemical Physics, Chernogolovka, USSR) USSR Inventor's Certificate 255221 (1967), Byull. Izobr. No. 10, 1971; French Patent 2088 668, 1972; US Patent 3 726 643, 1973; UK Patent 1 321,084, 1974; Jap. Patent 1098 839, 1982.

2. A.G. Merzhanov, I.P. Borovinskaya,. Self-spreading high-temperature synthesis of refractory compounds, Doklady Chemistry, 204 (1972) 429 - 431. 
3. A.G. Merzhanov, Self-propagating high-temperature synthesis: Twenty years of research and findings, in: Z. Munir, J.B. Holt (Eds.) Combustion and Plasma Synthesis of HighTemperature Materials, VCH, New York, 1990, pp. 1-53.

4. V. Hlavacek, Combustion synthesis: A historical perspective, Amer. Ceram. Soc. Bull., 70 (1991), Vol. 70, No. 2, pp. 240-243.

5. A.G. Merzhanov, History and Recent Development in SHS, Ceramic International, 21(5) (1995) 371-379.

6. A. Varma, A.S. Rogachev, A.S. Mukasyan, S. Hwang, , Combustion synthesis of advanced materials: Principles and applications, in: J. Wei (Ed.) Advances in Chemical Engineering, New York: Academic Press, 1998, Vol. 24, pp. 79-226.

7. A.S. Rogachev, A.S. Mukasyan, Combustion for Materials Synthesis, CRC Press, Taylor\& Francis, Boca Raton, London, New York, 2015.

8. A.G. Merzhanov, 40 years of SHS: A lucky Star of Scientific Discovery, Bentham Science, e-book, eISBN \#: 978-1-60805-128-1 (2012) p.112.

9. K.C. Patil, S.T. Aruna, T Mimani. Combustion synthesis: an update, Current Opinion in Solid State and Materials Science, 6 (2002) 507-512.

10. S.T. Aruna, A.S.Mukasyan. Combustion synthesis and Nanomaterials, Current Opinion in Solid State and Materials Science, 12 (2008) 44-50.

11. A.S.Rogachev, Exothermic reaction waves in multilayer nano-films, Russian Chemical Review, 77(1) (2008) 21-37.

12. A.S.Rogachev, A.S. Mukasyan, Combustion of heterogeneous nanostructural systems, Combustion Explosion and Shock Waves, 46(3) (2010) 21-28. 
13. T.P. Yadav, R.M. Yadav, D.P. Singh, Mechanical Milling: a top down approach for the synthesis of nanomaterials and nanocomposites, Nanoscience and Nanotechnology, 2 (2012) 22-48.

14. C. Suryanarayana, Mechanical Alloying and Milling. Marcel Dekker, New York, 2004.

15. V. Rajkovic, D. Bozic, M.T. Jouanovic, Properties of copper matrix with various reinforced size and amount of $\mathrm{Al}_{2} \mathrm{O}_{3}$ particles, Journal of Material Processing Technology, (1-3) (2008) 106-114.

16. N.-R. Park, D.-M. Lee, I.-Y. Ko, J.-K. Yoon, I.-J. Shon, Rapid consolidation of nanocrystalline $\mathrm{Al}_{2} \mathrm{O}_{3}$ reinforced $\mathrm{Ni}-\mathrm{Fe}$ composite from mechanically alloyed powders by high frequency induction heated sintering, Ceramics International, 35(8) (2009) $3147-3151$.

17. S. Sheibani, Manesh S. Heshmati,A. Ataie, Structural investigation on nano-crystalline $\mathrm{Cu}-\mathrm{Cr}$ supersaturated solid solution prepared by mechanical alloying, Journal of Alloys and Compounds, 495(1) (2010) 59-62.

18. J.M. Loureiro, B.F.O. Costa B, G. Le Caer, Mechanosynthesis of supersaturated solid solutions of Sn in near-equiatomic bcc FeCo, Journal of Alloys and Compounds, 536( 2012) S31-S34.

19. C.A. Schuh, T.C Hufnagel, R. Upadrasta, Overview No.144 - Mechanical behavior of amorphous alloys, Acta Materialia, 55(12) (2007) 4067-4109.

20. C. Politis, Bulk amorphous and nanostructured materials by high energy ball milling, International Journal of Modern Physics B, 22(18-19) (2008) 2905-2913. 
21. A. Molladavoudi, S. Amirkhanlou, M. Shamanian, F. Ashrafizadeh, The production of nanocrystalline cobalt titanide intermetallic compound via mechanical alloying, Intermetallics, 29 (2012) 104-109.

22. S. Sabooni, F. Karimzadeh, M.H. Abbasi, Thermodynamic aspects of nanostructured $\mathrm{Ti}_{5} \mathrm{Si}_{3}$ formation during mechanical alloying and its characterization, Bulletin of Materials Science, 35(3) (2012) 439-447.

23. T. Frolov, K.A. Darling, L.J. Kecskes, Y. Mishin, Stabilization and strengthening of nanocrystalline copper by alloying with tantalum, Acta Materialia, 60(5) (2012) $2158-2168$.

24. L Takacs, Self-sustaining reactions induced by ball milling, Progress in Materials Science, 47(4) (2002) 355-414.

25. E.L. Dreizin. Metal-based reactive nanomaterials, Progress in Energy and Combustion Science, 35 (2009) 141-167.

26. C.C. Koch, J.D. Whittenberger, Mechanical milling/alloying of intermetallics, Intermetallics, 4 (1996) 339-355.

27. T.F. Grigorieva, A.P. Barinova, N.Z Lyakhov, Mechanochemical synthesis in the metallic systems, Novosibirsk: Institute of solid state chemistry and mechanochemistry, Russian Academy of Sciences Publishing, 2008; ISBN 978-5-98901-050-9 (in Russian).

28. O.I. Lomovsky, Mechanocomposites - precursors for materials with new properties. Novosibirsk: Publishing House of the Siberian branch of the Russian Academy of Sciences, 2010, ISBN 978-5-7692-1108-9 (in Russian).

29. A.G. Ermilov, E.B Bogatyreva, Preliminary mechanoactivation, Moscow: Publishing house Moscow institute of steel and alloys, 2012, ISBN 978-5-87623-650-0 (in Russian). 
30. S. Rosenkranz, S. Breitung-Faes, W. Kwade, Experimental investigation and modeling of the ball motion in planetary ball mills, Powder Technology, 212 (2011) 224-230.

31. Y-S Kwon, K.B. Gerasimov, S-K Yoon, Ball temperatures during mechanical alloying in planetary mills, Journal of Alloys and Compounds , 346 (2002) 276-281.

32. J.Trapp, B. Kieback, Solid-state reactions during high-energy milling of mixed powders, Acta Materialia, 61 (2013) 310-320.

33. T. Grigorieva, M. Korchagin, N Lyakhov, Combination of SHS and mechanochemical synthesis for nanopowder technologies, KONA Powder and Particles, 20 (2002) $144-158$.

34. N.Z Lyakhov, T.L. Talako, T.F. Grigorieva, Influence of the mechanical activation on the phase- and structure formation at self-propagating high temperature synthesis, Novosibirsk: Parallel, 2008, ISBN 978-5-98901-051-6 (in Russian).

35. A.S. Mukasyan, B.B. Khina, R.V. Reeves, S.F Son, Mechanical activation and gasless explosion: nanostructural aspects, Chemical Engineering Journal, 174(2-3) (2001) $677-686$.

36. L.L. Shaw, Material processing via an integrated mechanical and thermal activation process, Materials and advanced manufacturing processes, 16(3) (2001) 405-418.

37. K.V. Manukyan, B.A. Mason, L.J. Groven, Y.-C Lin, M.Cherukara, S.Son, A. Strachan, A.S. Mukasyan, Tailored reactivity of $\mathrm{Ni}+\mathrm{Al}$ nanocomposites: microstructural correlations, Journal of Physical Chemistry C, 116 (39) (2012) 21027-21038.

38. A.S. Rogachev, N.F. Shkodich, S.G. Vadchenko, F. Baras, D. Yu Kovalev, S. Rouvimov, A.Nepapushev, A.S. Mukasyan, Influence of the high energy ball milling on structure 
and reactivity of the $\mathrm{Ni}+\mathrm{Al}$ powder mixture, Journal of Alloys and Compounds, 577 (2013) 600-605.

39. L. Thiers, A.S. Mukasyan, A. Varma, Thermal Explosion in Ni-Al System: Influence of Reaction Medium Microstructure, Combustion and Flame, 131 (1-2) (2002) 198-209.

40. J.D.E White, R.V. Reeves, S.F Son, A.S. Mukasyan, Thermal explosion in Al-Ni system: influence of mechanical activation, Journal of Physical Chemistry A, 113 (2009) 1354113547.

41. A.S. Shteinbertg, Y.-C. Lin, S.F. Son, A.S. Mukasyan, Kinetics of high temperature reaction in Ni-Al system: influence of mechanical activation, Journal of Physical Chemistry A, 114 (2010) 6111-6116.

42. A.S. Rogachev, N.A. Kochetov, N.V. Sachkova, V.V. Kurbatkina, E.A. Levashov, P.S. Grinchuk, O.S. Rabinovich, F. Bernard, Microstructural aspects of gasless combustion of mechanically activated mixtures: I. High-speed micro video recording of the Ni-Al composition, Combustion Explosion and Shock Waves, 42(4) (2006) 421-429.

43. A.S. Mukasyan, J.D.E. White, D.Y. Kovalev, N.A. Kochetov, V.I Ponomarev, S.F. Son, Dynamics of phase transformation during thermal explosion in the Al-Ni system: influence of mechanical activation, Physica B-Condensed Matter, 405 (2010) 778-784.

44. K.V. Manukyan, Ya-C Lin, S. Rouvimov, P.J McGinn, A.S. Mukasyan, Microstructurereactivity relationship of Ti-C reactive nanomaterials, Journal of Applied Physics, 113 (2) (2013) 024302-024310.

45. A.S. Mukasyan, Combustion synthesis of silicon carbide: Properties and Applications of Silicon Carbide, in: R. Gerhardt (Ed.), Janeza Trdine: InTech; 2011, pp. 389-409. 
46. R. Pampuch, L. Stobierski, J. Liz, M. Raczka, Solid combustion synthesis of $\beta$-SiC powder, Material Research Bulletin, 22 (1987) 1225-1231.

47. O. Yamada, Y. Miyamoto, M Koizumi, Self-propagating high-temperature synthesis of SiC, Journal of Material Research, 1(2) (1986) 275-279.

48. H. Xue, Z.A. Munir, The synthesis of composites and solid solutions of $\alpha$-SiC-AlN by field-activated combustion, Scripta Materialia 35(8) (1996) 979-982.

49. Z.A. Munir, Field effects in self-propagating solid-state synthesis reactions, Solid State Ionics, 101-103 (1997) 991-1001.

50. S. Gedevanishvili, Z.A Munir, An investigation of the combustion synthesis of $\mathrm{MoSi}_{2} \beta-$ $\mathrm{SiC}$ composites through electric-field activation, Material Science and Engineering A, $242(1-2)(1998) 1-6$.

51. G.A. Nersisyan, V.N. Nikogosov, S.L. Kharatyan, A.G. Merzhanov, Chemical transformation mechanism and combustion regimes in the system silicon-carbonfluoroplast, Combustion, Explosion and Shock Waves, 27(6) (1991) 720-724.

52. A.S. Mukasyan, A.G. Merzhanov, V.M. Martinenko, I.P. Borovinskaya, M.Y. Blinov, Mechanism and principles of silicon combustion in nitrogen, Combustion, Explosion and Shock Waves, 22 (5) (1986) 534-540.

53. Y. Yang, K. Yang, Z-M Lin, J-T Li, Mechanical-activation-assisted combustion synthesis of SiC, Materials Letters, 61 (2007) 71-76.

54. Y. Yang, K. Yang, Z-M Lin, J-T Li, Synthesis of SiC by silicon and carbon combustion in air, Journal of the European Ceramic Society, 29 (2009) 175-180. 
55. A.S. Mukasyan, Ya-C Lin, A.S. Rogachev, D.O. Moskovskikh, Direct combustion synthesis of silicon carbide nanopowder from the elements, Journal of the American Ceramic Society, 96(1) (2013) 111-117.

56. D.O. Moskovskikh, A.S. Mukasyan, A.S. Rogachev, Self-propagating high-temperature synthesis of silicon carbide nanopowders, Doklady Physical Chemistry, 449 (2013) 4143.

57. S.T. Aruna, Solution Combustion Synthesis-An Overview, in M. Lackner (Ed.) Combustion Synthesis: Novel Routes to Novel Materials, Bentham Publishers, 2010, pp. 206-221.

58. Y. Liu, Y. Jiang, X. Zhang, Y. Wang Y, Y. Zhang, H. Liu, H. Zhai, Y. Liu, J. Yang, Y. Yan, Effects of annealing temperature on the structure and magnetic properties of the L10-FePt nanoparticles synthesized by the modified sol-gel method, Powder Technology, 239 (2013) 217-222.

59. T. Minami, Advanced sol-gel coatings for practical applications, Journal of Sol-Gel Science and Technology 65 (1) (2013) 4-11.

60. A. Sutka, G. Mezinskis, Sol-gel auto-combustion synthesis of spinel-type ferrite nanomaterials, Frontiers of Material Science, 6(2) ( 2012) 128-141.

61. S.I. Roslyakov, D Yu Kovalev, A.S. Rogachev, K. Manukyan, A.S. Mukasyan, Solution Combustion: Dynamic of phase transformation during synthesis of high porous nickel, Doklady Physical Chemistry, 449 (2013) 48-51.

62. R.K. Selvan, C.O. Augustin, L.J Berchmans, R. Saraswathi, Combustion synthesis of $\mathrm{CuFe}_{2} \mathrm{O}_{4}$, Materials Research Bulletin. 38(1) (2003) 41-54. 
63. C-C Hwang, J-S Tsai , T-H Huang, Combustion synthesis of Ni-Zn ferrite by using glycine and metal nitrates - investigations of precursor homogeneity, product reproducibility, and reaction mechanism, Materials Chemistry and Physics, 93(2-3) (2005) 330-336.

64. P. Vijaya Bhasker Reddy, B. Ramesh, C. Gopal Reddy, Electrical conductivity and dielectric properties of zinc substituted lithium ferrites prepared by sol-gel method, Physica B: Condensed Matter, 405(7) (2010) 1852-1856.

65. V. Sreeja, S. Vijayanand, S. Deka et al. Magnetic and Mössbauer spectroscopic studies of NiZn ferrite nanoparticles synthesized by a combustion method, Hyperfine Interactions, 183(1-3) (2008) 99-107.

66. K.H.Wu, C.H. Yu, Y.C. Chang et al. Effect of $\mathrm{pH}$ on the formation and combustion process of sol-gel auto-combustion derived $\mathrm{NiZn}$ ferrite/ $\mathrm{SiO}_{2}$ composites, Journal of Solid State Chemistry 177(11) (2004) 4119-4125.

67. L. Yu, S. Cao, Y Liu et al. Thermal and structural analysis on the nanocrystalline NiCuZn ferrite synthesis in different atmospheres, Journal of Magnetism and Magnetic Material, 301(1) (2006) 100-106.

68. M. Sertkol, Y. Kōseoglu, A. Baykal, H. Kavas, M.S. Toprak, Synthesis and magnetic characterization of $\mathrm{Zn}{ }_{0.7} \mathrm{Ni}_{0.3} \mathrm{Fe}_{2} \mathrm{O}_{4}$ nano particles via microwave assisted combustion route, Journal of Magnetism and Magnetic Material, 322(7) (2010) 866-871.

69. P. Erri, J. Nader, A. Varma, Controlling combustion wave propagation for transition metal/alloy/cermet foam synthesis, Advanced Materials, 20 (2008) 1243-1245. 
70. A. Kumar, E. E. Wolf, A. S. Mukasyan, Solution combustion synthesis of metal nanopowders: nickel—reaction pathways, American Institute of Chemical Engineers Journal , 57(8) (2011) 2207-2214.

71. A. Kumar, E. E. Wol, A. S. Mukasyan, Solution combustion synthesis of metal nanopowders: Copper and copper/nickel alloys, American Institute of Chemical Engineers Journal, 57(12) (2011) 3473-3479.

72. B.C. Tappan, S.A. Steiner III, E.P. Luther, Nanoporous metal foams, Angewandte Chemie International Edition, 49 (2010) 4544-4565.

73. B.C. Tappan, M.H. Huynh, M.A. Hiskey, D.E Chavez, E.P. Luther, J.T Mang, S.F. Son, Ultralow-density nanostructured metal foams: Combustion synthesis, morphology, and composition, Journal of American Chemical Society, 128 (2006) 6589-6594.

74. D.E. Chavez, M.A Hiskey, D.I. Naud , J. Pyrotech, The utility of 3,6-dihydrazino-1,2,4,5tetrazine (DHT) in indoor pyrotechnics, 24-th Intern Pyrotech Seminar (1999) 161-177.

75. M. Friedrich, J.C. Galvez-Ruiz, T.M. Klapötke, P. Mayer, B. Weber, J.J Weigand , BTA copper complexes, Inorganic Chemistry, 44 (2005) 8044-8052.

76. J. Stierstorfer, K.R. Tarantik, T.M. Klapötke, New energetic materials: functionalized 1ethyl-5-aminotetrazoles and 1-Ethyl-5-nitriminotetrazoles, European Journal of Chemistry, 15 (2009) 5775-5792.

77. E.M. Hunt, M.L. Pantoya, R.J. Jouet, Combustion synthesis of metallic foams from nanocomposite reactants, Intermetallics, 14 (2006) 620-629.

78. Z. Hua, Y. Deng, K. Li K, S.Yang, Low-density nanoporous iron foams synthesized by sol-gel autocombustion, Nanoscale Research Letters, 7(1) (2012) 1-7. 
79. E. Pál, R. Kun, C. Schulze, V. Zöllmer, D. Lehmhus, M. Bäumer, M. Busse, Composition-dependent sintering behaviour of chemically synthesised $\mathrm{CuNi}$ nanoparticles and their application in aerosol printing for preparation of conductive microstructures, Colloid and Polymer Science, 290 (2012) 941-952.

80. A.Cross, A. Kumar, E.E Wolf, A.S. Mukasyan, Combustion synthesis of a nickel supported catalyst: Effect of metal distribution on the activity during ethanol decomposition, Industrial and Engineering Chemistry Research, 51 (2012) $12004-12008$.

81. V.M. Shinde, G. Madras, Kinetic studies of ionic substituted copper catalysts for catalytic hydrogen combustion, Catalysis Today, 198 (2012) 270- 279.

82. J.L. Carlos, J.R De la Rosa, H.R. Aracely, M.L. Enrique, B.P. Georgina, M.G. Rosa del Carmen, D.P.S Celia, La-, Mn- and Fe-doped zirconia catalysts by sol-gel synthesis: TEM characterization, mass-transfer evaluation and kinetic determination in the catalytic combustion of trichloroethylene, Colloids and Surfaces A: Physicochemical Engineering Aspects, 371 (2010) 81-90.

83. C.H. Han, H.S. Lee, S.D. Han, Synthesis of Nanocrystalline $\mathrm{TiO}_{2}$ by Sol-Gel Combustion Hybrid Method and Its Application to Dye Solar Cells, Bulletin of the Korean Chemical Society 29(8) (2008) 1495-1498.

84. Gangulibabu, B. Bhuvaneswari, N. Kalaiselvi, Comparison of corn starch-assisted solgel and combustion methods to prepare $\mathrm{LiMn}_{\mathrm{x}} \mathrm{Co}_{\mathrm{y}} \mathrm{Ni}_{\mathrm{z}} \mathrm{O}_{2}$ compounds, Journal of Solid State Electrochemistry, 17 (2013) 9-17. 
85. Y. Yuan, C. Liu, Y. Zhang, X. Shan, Sol-gel auto-combustion synthesis of hydroxyapatite nanotubes array in porous alumina template, Materials Chemistry and Physics, 112 (2008) 275-280.

86. H.F. Spedding, A.H. Wilhelm, H.W. Keller (United States Atomic Energy Commission) US patent 2830894, 1958.

87. L. Wang, D. Luss, K.S. Martirosyan, The behavior of nanothermite reaction based on $\mathrm{Bi}_{2} \mathrm{O}_{3} / \mathrm{Al}$, Journal of Applied Physics, 110 (2011) 074311.

88. K.S. Martirosyan, L. Wang, Vicent A, D. Luss, Synthesis and performance of bismuth trioxide nanoparticles for high energy gas generator use, Nanotechnology, 20 (2009) 405609.

89. C.D. Yarrington, S.F. Son, T.J Foley, S.J. Obrey, A.N. Pacheco, Nano aluminum energetics: the effect of synthesis method on morphology and combustion performance, Propellants Explosion Pyrotechnics, 36 (2011) 551 - 557.

90. J.L Cheng, H.H Hng, H.Y Ng, P.C. Soon, Y.W. Lee, Synthesis and characterization of self-assembled nanoenergetic $\mathrm{Al}-\mathrm{Fe}_{2} \mathrm{O}_{3}$ thermite system, Journal of Physical Chemistry Solids, 71(2) (2010) 90-94.

91. J.L. Cheng, H.H Hng, Y.W. Lee, S.W. Du, N.N Thadhani, Kinetic study of thermal- and impact-initiated reactions in $\mathrm{Al}-\mathrm{Fe}_{2} \mathrm{O}_{3}$ nanothermite, Combustion and Flame 157 (2010) 2241-2249.

92. B. Siegert, M. Comet, O. Muller, G. Pourroy, D. Spitzer, Reduced-sensitivity nanothermites containing manganese oxide filled carbon nanofibers, Journal of Physical Chemistry C 114 (2010) 19562-19568. 
93. J.J. Granier, M.L. Pantoya, Laser ignition of nanocomposite thermites, Combustion and Flame, 138 (2004) 373-383.

94. A.S. Rogachev, A.S. Mukasyan, Discrete Reaction Waves: Gasless Combustion of Solid Powder Mixtures, Journal of Progress in Energy and Combustion Science, 34(3) (2008) 377-416.

95. V.I. Rozenband, N.I. Vaganova NI, A strength model of heterogeneous ignition of metal particles, Combustion and Flame, 88 (1992) 113-118

96. V. Rosenband, Thermo-mechanical aspects of the heterogeneous ignition of metals, Combustion and Flame, 137 (2004) 366-375.

97. K. Park, D. Lee, A. Rai, D. Mukherjee, M.R. Zachariah, Size-resolved kinetic measurements of aluminum nanoparticle oxidation with single particle mass spectrometry, Journal of Physical Chemistry B, 109 (2005) 7290-7299.

98. A. Rai, K. Park, L. Zhou, M.R. Zachariah, Understanding the mechanism of aluminium nanoparticle oxidation, Combustion Theory and Modelling, 10(5) (2006) 843-859.

99. V.I. Levitas, B.W. Asay, S.F. Son, M.L. Pantoya, Mechanochemical mechanism for fast reaction of metastable intermolecular composites based on dispersion of liquid metal, Journal of Applied Physics, 101 (2007) 083524.

100. V.I. Levitas, M.L. Pantoya, B. Dikici, Melt-dispersion mechanism for fast reaction of aluminum particles: Extension for micron scale particles and fluorination, Applied Physics Letters, 92 (2008) 011921.

101. K.W. Watson, M.L. Pantoya, V.I. Levitas, Fast reactions with nano- and micrometer aluminum: a study on oxidation versus fluorination, Combustion and Flame, 155 (2008) 619-634. 
102. M.A Trunov, M. Schoenitz, E.L Dreizin, Effect of polymorphic phase transformations in alumina layer on ignition of aluminium particles, Combustion Theory and Modelling, 10(4) (2006) 603-623.

103. M.A. Trunov, S.M. Umbrajkar, M. Schoenitz, J.T. Mang, E.L. Dreizin, Oxidation and melting of aluminum nanopowders, Journal of Physical Chemistry B, 110 (2006) 13094-13099.

104. A.S. Mukasyan, V.A Shugaev, N.B Kiryakov, The Influence of Gaseous Fluid Phase on Combustion of Titanium in Air, Combustion Explosion and Shock Waves, 29(1) (1993) 7-11.

105. H. Goldschmidt, (Chemische Fabrik Th), Deutsche Reichs Patent no. 96317, 13 March 1895.

106. E. Collins, M. Pantoya, W. Vijayasai, T. Dallas, Comparison of engineered nanocoatings on the combustion of aluminum and copper oxide nanothermites, Surface and Coatings Technology, 215 (2013) 476-484.

107. K.T. Higa, Journal of Energetic nanocomposite lead-free electric primers, Journal of Propulsion and Power, 23 (2007) 722-727.

108. S.J Apperson, A.V. Bezmelnitsyn, R. Thiruvengadathan , K. Gangopadhyay, S. Gangopadhyay, W.A. Balas, P.E. Anderson, S.M. Nicolich, Characterization of nanothermite material for solid-fuel microthruster applications, Journal of Propulsion and Power, 25(5) (2009) 1086-1091.

109. S. Apperson, R.Shende, S. Subramanian, D. Tappmeyer, S. Gangopadhyay, Z. Chen, K. Gangopadhyay, P. Redner, S. Nicholich, D. Kapoor, Generation of fast 
propagating combustion and shock waves with copperoxide / aluminum nanothermite composites, Applied Physics Letters, 91(24) (2007) 243109.

110. C. Rossi, K. Zhang, D. Esteve, P. Alphonse, P. Tailhades, C.J. Vahlas, Nanoenergetic materials for MEMS: A review, Journal of Microelectromechanical System, 16 (2007) 919-931.

111. V. Pichot, M. Comet, E. Fousson , C. Baras, A. Senger, F. Le Normand, D. Spitzer, An efficient purification method for detonation nanodiamonds, Diamond and Related Material, 17 (2008) 13-22.

112. K.S. Martirosyan, L. Wang, A. Vicent,D. Luss, Nanoenergetic gas-generators: design and performance, Propellants Explosion and Pyrotechnics, 34 (2009) 532 - 538.

113. T.P. Weihs, Self-propagating reactions in multilayer materials in: D.A. Glocker, S.I. Shah (Eds.), Handbook of thin films process technology, Institute of Physics Publishing, Bristol, Part F, 1998.

114. M.E. Reiss, C.M. Esber, D. Van Heerden, A.J. Gavens, M.E. Williams, T.P. Weihs, Self-propagating formation reactions in $\mathrm{Nb} / \mathrm{Si}$ multilayers, Materials Science and Engineering, A261 (1999) 217-222.

115. R.V. Reeves, M.A. Rodrigues, E.D. Jones, D.P. Adams, Condenced -phase and oxidation reaction behavior of $\mathrm{Ti} / 2 \mathrm{~B}$ foils in various gaseous environments, The Journal of Physical Chemistry C, 116 (2012) 17904-17912.

116. A.K. Stover, N.K. Walker, R. Knepper, G.M. Fritz, T.C. Hufnagel, T.P Weihs, Correlating mechanical properties to microstructure in rolled nickel aluminum reactive laminates, Boston, JANNAF Conference, 2008. 
117. C.T. Wei, B.R. Maddox, A.K. Stover, T.P. Weihs, V.F. Nesterenko, M.A. Meyers Reaction in Ni-Al laminates by laser-shock compression and spalling, Acta Materialia, 59 (2011) 5276-5287.

118. P.E. Specht, N.N. Thadhani, T.P. Weihs, Configuration effects on shock wave propagation in Ni-Al multilayer composites, Journal of Applied Physics, 111 (2012) 073527.

119. V.C. Srivastava, T. Singh, Ghowdhury Ghosh, V. Jindal, Microstructural characteristics of accumulative roll-bonded $\mathrm{Ni}-\mathrm{Al}$ - based metal-intermetallic laminate composite. Journal of Materials Engineering and Performance, 21(9) (2012) 1912-1918.

120. C.T. Wei, V.F. Nesterenko, T.P. Weihs, B.A. Remington, H.S. Park, M.A. Meyers, Response of Ni/Al laminates to laser-driven compression, Acta Materialia, 60 (2012) 3929-3942.

121. A. Mozaffari, H. Danesh Manesh, K. Janghorban, Evaluation of mechanical properties and structure of multilayered $\mathrm{Al} / \mathrm{Ni}$ composites produced by accumulative roll bonding (ARB) process, Journal of Alloys and Compounds, 489 (2010) 103-109.

122. A. Mozaffari, M. Hosseini, H. Danesh Manesh, Al/Ni metal intermetallic composite produced by accumulative roll bonding and reaction annealing, Journal of Alloys and Compounds, 509 (2011) 9938 -9945.

123. M.I. Karpov, V.I Vnukov, K.G. Volkov, N.V. Medved, I.I. Khodos I, G.E. Abrosimova, Possibilities of the vacuum rolling as method for production of multilayer composites with nanometric thicknesses of the layers, Materialovedenie, , 1 (2004) 4853 (in Russian). 
124. B.S. Bokstein, V.I. Vnukov, E.V. Golosov, M.I. Karpov, Yu.R. Kolobov, D.A. Kolesnikov, V.P. Korzhov, A.O. Rodin, Structure and diffusion properties in laminated composites of a Cu-Ti system, Russian Physics Journal, 52(8) (2009) 811-815.

125. V.I. Betekhin, Yu.R. Kolobov, B.K. Kardashev, E.V. Golosov, M.V. Narykova, A.G. Kadomtsev, D.N. Klimenko, M.I Karpov, Elasto-plastic properties of $\mathrm{Cu}-\mathrm{Nb}$ Nanolaminate, Technical Physics Letters, 18(2) (2012) 144-146.

126. M.I Karpov, V.P. Korzhov, V.I. Vnukov, Yu.R. Kolobov, E.V. Golosov, Structure and hardness of multilayer nanostructural $\mathrm{Cu} / \mathrm{Ag}$-composite, Tambov University Reports (Vestnik TGU), 15(3) (2010) 941-942 (in Russian).

127. V.P. Korzhov, M.I Karpov, A.N. Nekrasov, Structure and hardness of multilayer Ti/Ni-microcomposite produced by rolling. Tambov University Reports (Vestnik TGU), 15(3) (2010) 945-946 (in Russian).

128. M.I Karpov, V.P. Korzhov, V.M. Kiiko, A.N. Nekrasov, Structure of plane composites on nickel base reinforced by intermetallic layers, Tambov University Reports (Vestnik TGU). 15(3) (2010) 947-948 (in Russian).

129. S.G. Vadchenko, A.S. Rogachev, Method for production of multilayer energetic nanostructured films for the permanent connection of metals, Russian Patent Application 2012107273/02(011058), 29.02.2012; Positive Decision 28.11.2012 (in Russian).

130. E. Ma, C.V. Thompson, L.A. Clevenger, Nucleation and growth during reactions in multilayer Al/Ni films: The early stage of Al, Ni formation, Journal of Applied Physics 69(4) (1991) 2211-2218. 
131. C. Michaelsen, K. Barmak, Calorimetric determination of $\mathrm{NiAl}_{3}$-growth kinetics in sputter-deposited Ni/Al diffusion couples, Journal of Alloys and Compounds, 257 ( 1997) 211-214.

132. J.C. Trenkle, L.J. Koerner, M.W. Tate, Noel Walker, S.M. Gruner, T.P. Wihs, T.C. Hufnagel, Time-resolved x-ray microdiffraction studies of phase transformations during rapidly propagating reactions in $\mathrm{Al} / \mathrm{Ni}$ and $\mathrm{Zr} / \mathrm{Ni}$ multilayer foils, Journal of Applied Physics 107 (2010) 113511.

133. M. Vohra, M. Grapes, P. Swaminathan, T.P. Weihs, O.M. Knio, Modeling and quantitative nanocalorimetric analysis to assess interdiffusion in a Ni/Al bilayer, Journal of Applied Physics, 110 (2011) 123521.

134. J.C. Crone, J. Knap, P.W. Chung, B.M. Rice, Role of microstructure in initiation of Ni-Al reactive multilayers, Applied Physics Letters, 98 (2011) 141910.

135. A.B. Mann, A.J. Gavens, M.E. Reiss, D. Van Heerden, G.Bao, T.P. Weihs, Modeling and characterizing the propagation velocity of exothermic reactions in multilayer foils, Journal of Applied Physics, 82(3) (1997) 1178-1188.

136. St. Frank, S.V. Divinski, U. Sodervall, Chr. Herzig, Ni tracer diffusion in the B2compound NiAl: influence of temperature and composition, Acta Materialia, 49 (2001) 1399-1411.

137. A.V. Evteev, E.V. Levchenko, I.V. Belova, G.E. Murch, Molecular dynamics , simulation of diffusion in a (110) B2-NiAl film, Intermetallics, 19 (2011) 848-854.

138. S. Stuber, D. Holland-Moritz, T. Unruh, A. Meyer, Ni self-diffusion in refractory Al-Ni melts, Physical Review B, 81 (2010) 024204. 
139. A.S. Rogachev, S.G. Vadchenko, A.S. Mukasyan, Self-sustained waves of exothermic dissolution in reactive multilayer nano-foils, Applied Physics Letters, 101 (2012) 063119.

140. O. Politano, F. Baras, A.S. Mukasyan, S.G. Vadchenko, A.S. Rogachev, Microstructure development during $\mathrm{NiAl}$ intermetallic synthesis in reactive $\mathrm{Ni}-\mathrm{Al}$ nanolayers: Numerical investigations vs. TEM observations, Surface and Coatings Technology 215 (2013) 485-492.

141. X. Qiu, R. Liu, S. Guo, J.H. Graeter, L. Kecskes, J. Wang, Combustion synthesis reactions in cold-rolled $\mathrm{Ni} / \mathrm{Al}$ and $\mathrm{Ti} / \mathrm{Al}$ multilayers, Metallurgical and Materials Transactions A, 40 (2009) 1541-1546.

142. B. Boettge, J. Braeuer, M. Wiemer, M. Petzold, J. Bagdahn, T. Gessner, Fabrication and characterization of reactive nanoscale multilayer systems for lowtemperature bonding in microsystem technology, Journal of Micromechanics and Microengineering, 20 (2010) 064018.

143. M.D. Wiemer, J. Braeuer, D. Wünsch, T. Gessner, Reactive bonding and low temperature bonding of heterogeneous materials, ECS Transactions 33(4) (2010) $307-318$.

144. A.S. Ramos, M.T. Vieira, Intermetallic compound formation in Pd/Al multilayer thin films, Intermetallics, 25 (2012) 70-74.

145. J. Braeuer, J. Besser, M. Wiemer, T. Gessner, A novel technique for MEMS packaging: Reactive bonding with integrated material systems, Sensors and Actuators A 188 (2012) 212-219. 
146. X. Zhou, R. Shen, Y. Ye, P. Zhu, Y. Hu, L. Wu, Influence of Al/Cu reactive multilayer films additives on exploding foil initiator, Journal of Applied Physics, 110 (2011) 094505.

147. X. Qiu, R. Tang, R. Liu, H. Huang, S. Guo, H. Yu, A micro initiator realized by reactive Ni/Al nanolaminates, Journal of Material Science: Materials in Electronics, 23 ( 2012) 2140-2144.

148. S.C. Kelly, S. Barron, N. Thadhani, T.P. Weihs, Laser-accelerated flyer system for investigating reactions in Ni-Al mixtures, AIP Conference Proceedings, 1426 (2012) 599-602.

149. H. Joress, S.C. Barron, K.J.T. Livi, N. Aronhime, T.P. Weihs, Self-sustaining oxidation initiated by rapid formation reactions in multilayer foils, Applied Physics Letters, 101 (2012) 111908.

150. A. Kumar, A.S. Mukasyan and E.E. Wolf, Modeling impregnated layer combustion synthesis of catalysts for hydrogen generation from oxidative reforming of methanol, Industrial and Engineering Chemistry Research, 49 (2010) 11001-11008.

151. E.M. Lennon, M.C. Tanzy, V.A. Volpert, A.S. Mukasyan, A. Bayliss, Combustion of reactive solutions impregnated into a cellulose carrier: Modeling of two combustion fronts, Chemical Engineering Journal, 174 (1) (2011) 333-340.

152. Z. A. Munir, U. Anselmi-Tamburini, Self-propagating exothermic reactions: The synthesis of high-temperature materials by combustion, MATEC Science Reports, 3,277 (1989). 


\section{Figure Captions}

Figure 1: Schematics of self-ignition process in Ni-Al system (adopted Ref. [ 39 ])

Figure 2: SEM images of initial Ni-Al powder mixture (a) and after (b) and BF TEM images of HEBM Ni-Al powder mixture (c,d) :(Ni dark, Al white regions (adopted Ref. [37, 38])

Figure 3: Calculated correlation between content of activated nano-structures and temperature of the reaction initiation (adopted Ref. [38]).

Figure 4: Microstructure of the initial compacted sample and that after combustion reaction (adopted Ref. [37]).

Figure 5: The typical microstructures: Initial Ti particles and $\mathrm{C}$ flakes (a); same mixture after HEBM (b); cross section of the composite Ti/C particles (c); TiC powders obtained by CS of ball-milled composite particles (adopted Ref. [44]).

Figure 6: Typical microstructures of: Initial $\mathrm{Si}+\mathrm{C}$ mixture (a); same mixture after HEBM (b);(c,d) SiC powder after combustion synthesis (adopted Ref. [55]).

Figure 7. Typical temperature-time profiles for SCS in $\left(\mathrm{Ni}\left(\mathrm{NO}_{3}\right)_{2} \cdot 6 \mathrm{H}_{2} \mathrm{O}\right)$ /glycine $\left(\mathrm{C}_{2} \mathrm{H}_{5} \mathrm{NO}_{2}\right)$ system for gels prepared under different drying conditions (adopted Ref. [61]).

Figure 8. (a-c) IR images of the reaction front propagation in sol-gel nickel nitrate)/glycine system; (d) Temperature time distribution in the reaction front (adopted Ref. [61]).

Figure 9: Photograph of iron foam next to Fe-BTA unburnt pellet and in-set shows SEM data of iron foam in $100 \mathrm{~nm}$ scale (adopted Ref. [72]).

Figure 10: Photograph of (a) the iron precursor gel in a quartz tube before combustion and (b) the porous iron foam after combustion (adopted Ref. [78]).

Figure 11: SEM image of a printed, non-sintered van der Pauw structure (adopted Ref. [79]).

Figure 12: TEM image of ZLF showing the relatively large cavity sizes (adopted Ref. [82]).

Figure 13: TEM images of Al nanoparticles oxide layer following different exposure time in air: (a) 0 min; (b) 5 min; (c) 20 min; and (d) 90 min and (e) $\mathrm{Bi}_{2} \mathrm{O}_{3}+\mathrm{nAl}$ mixture (adopted Ref. [87]).

Figure 14: FESEM (a,c) and TEM (b,d) images of $\mathrm{Fe}_{2} \mathrm{O}_{3}-\mathrm{nAl}$ reactive mixtures prepared by selfassembled (a,b) and physical solvent mixing (c,d) methods. (adopted Ref. [91]). 
Figure 15: Schematic representation of the microstructure: (a) conventional mixture of spherical nanoparticles. (b) the oxide (red) phase is enclosed in a carbon nanotube (c) and actual TEM image of $\mathrm{MnO}_{2}$ particles inside CNTs (adopted ref.[92]).

Figure 16: Pressure profile inside an oxide-coated Al particle: (a) with oxide coating thickness less $<1 \mathrm{~nm}$; (b) while the right side is for particle (b) with coating thickness $>>1 \mathrm{~nm}$ (adopted Ref. [96]).

Figure 17: Diagram shows how nAl particles may react via a melt-dispersion mechanism. Initially melting of $\mathrm{Al}$ creates internal pressures followed by spallation of the oxide shell exposing the molten core and generating a pressure wave which disperses small $\mathrm{Al}$ clusters. (adopted Ref. [100]).

Figure 18: Change in mass of the aluminium powder oxidizing in a thermal analyzer. Different stages of oxidation are indicated and the respective changes in the growing alumina scale are shown schematically (adopted Ref. [103]).

Figure 19: Examples of the lamellar structures Ni/Al, produced by various methods:(a), (b) magnetron deposition; (c) - initial stack of the foils before cold rolling [121], (d) - after cold rolling [120]; (e),(f) - high-energy ball mill plus cold rolling.(a),(b),(f) - TEM, light phase Al, dark phase Ni; (c),(d),(e) - SEM, dark phase Ni, light phase Al.

Figure 20: Mechanical methods for production of the lamellar reaction nano-foils: hot pressing of the stacked foils + cold rolling (a) and HEBM of powder mixtures + cold rolling (b). 
Table 1. Some Properties of nAl Powders

\begin{tabular}{lllll}
\hline Powder & $\begin{array}{l}\text { \% of active } \\
\text { metal }\end{array}$ & $\begin{array}{l}\text { Nominal size } \\
\mathrm{nm}\end{array}$ & $\begin{array}{l}\text { Surface area } \\
\mathrm{m}^{2} / \mathrm{g}\end{array}$ & Supplier \\
\hline Novacentrix Al & 79 & 80 & 28 & \\
ALEX & 86 & 100 & 13 & Novacentrix \\
& & Argonide Corp \\
\hline
\end{tabular}




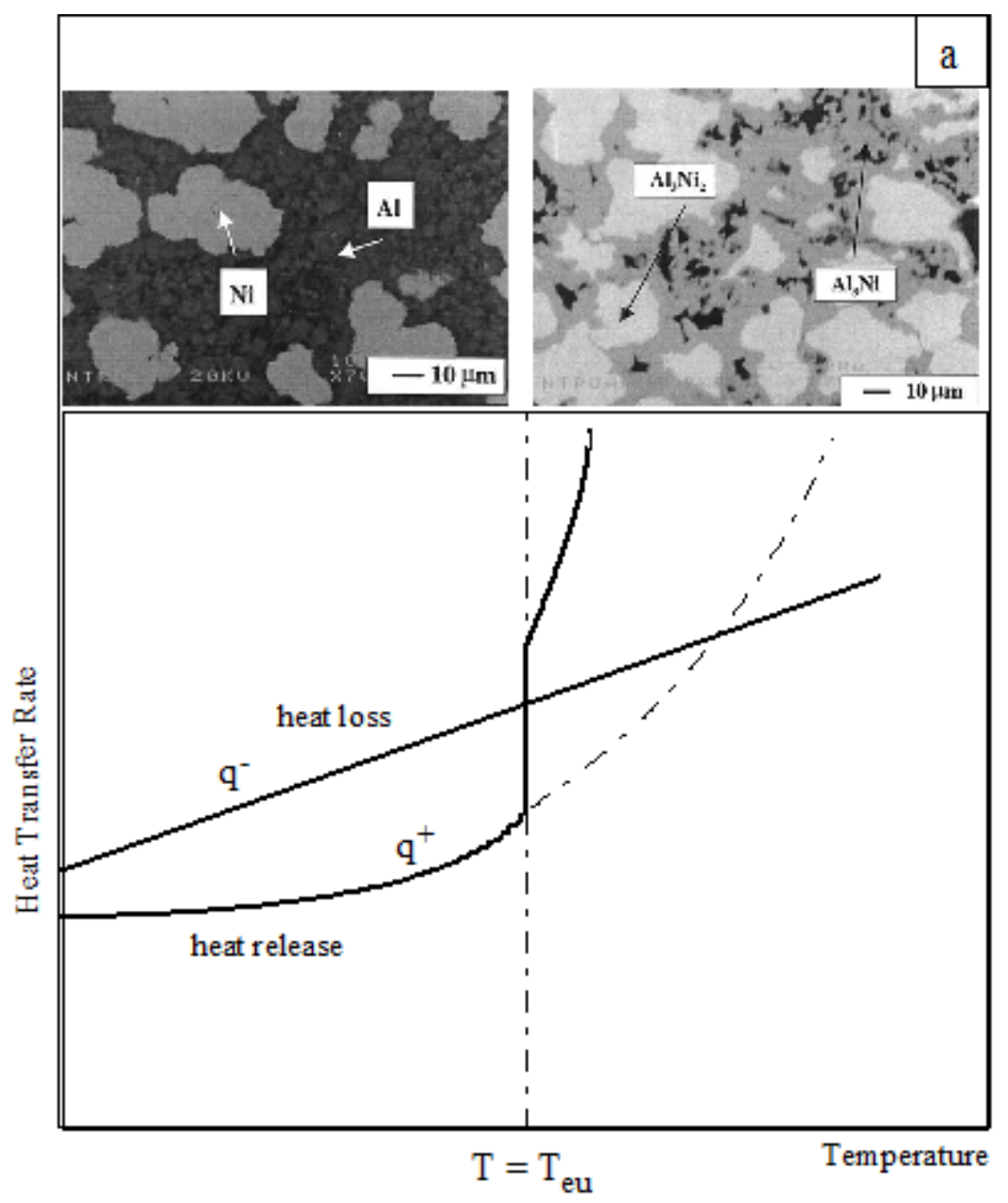

Figure 1 

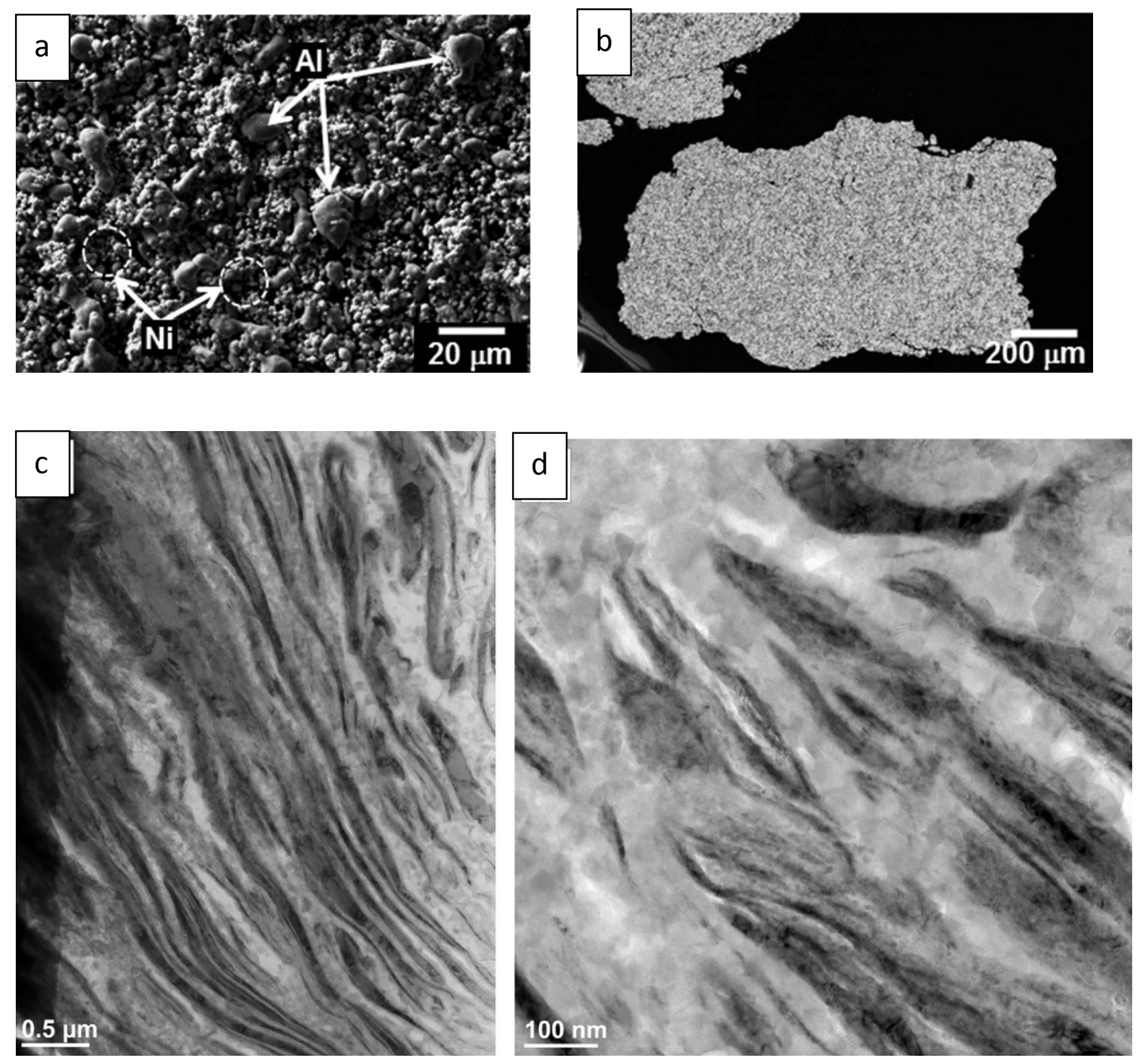

Figure 2 


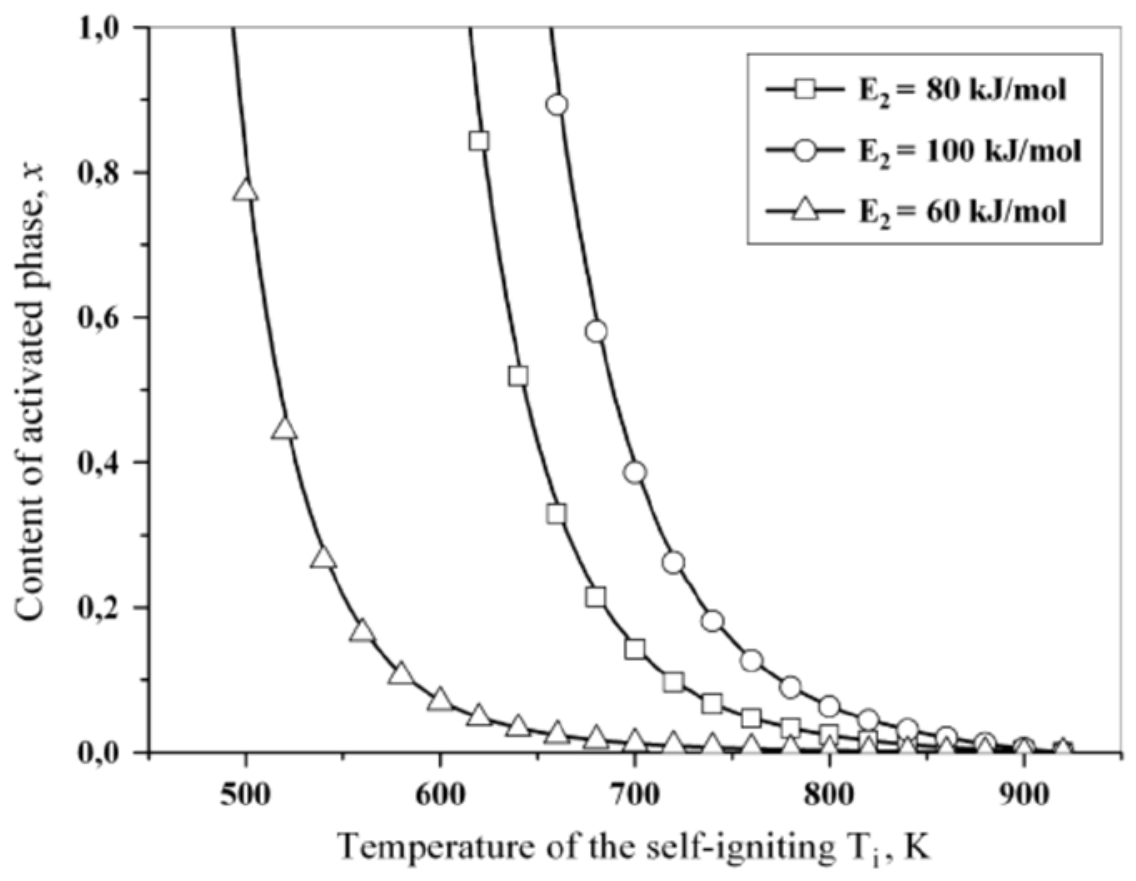

Figure 3 

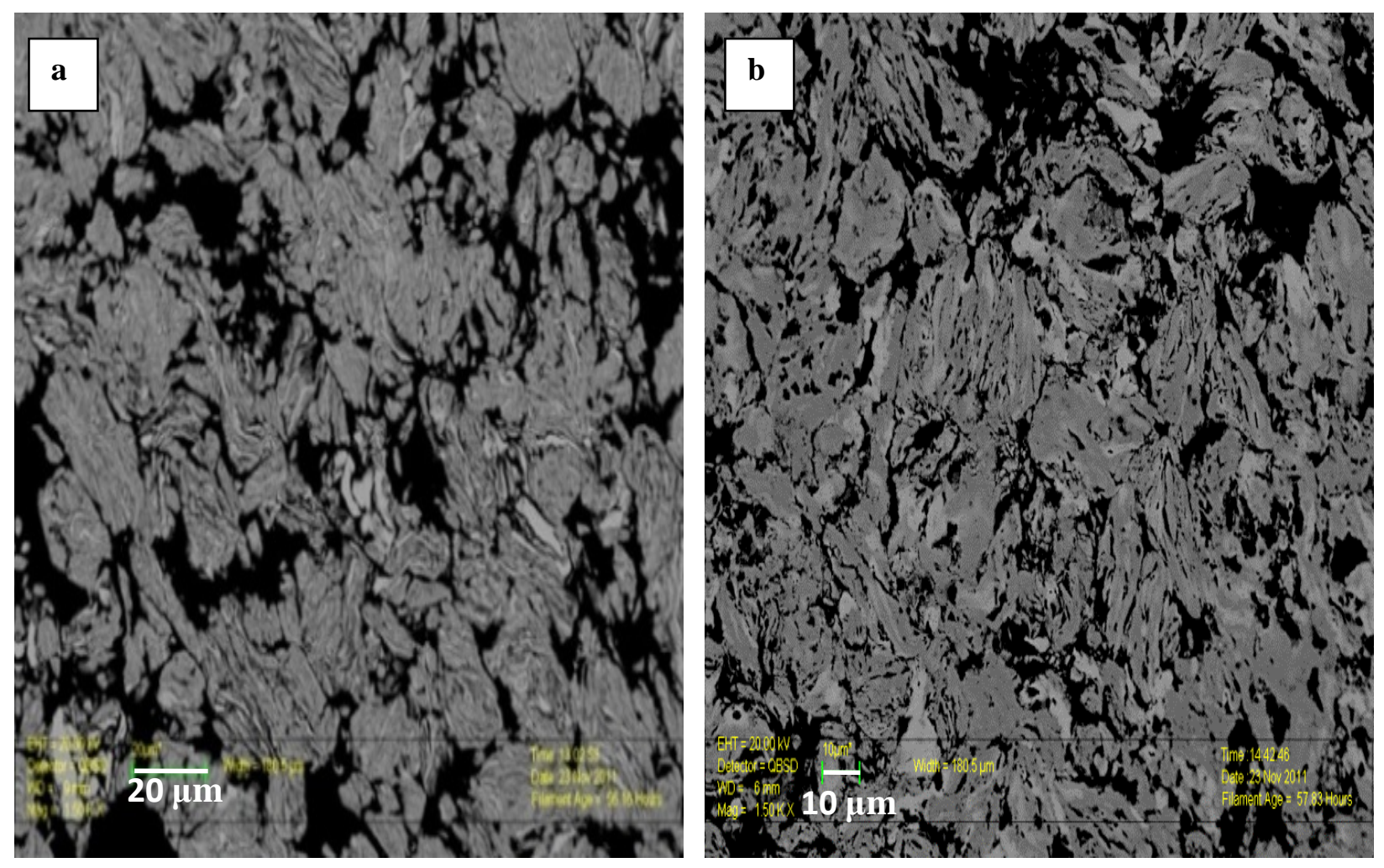

Figure 4 

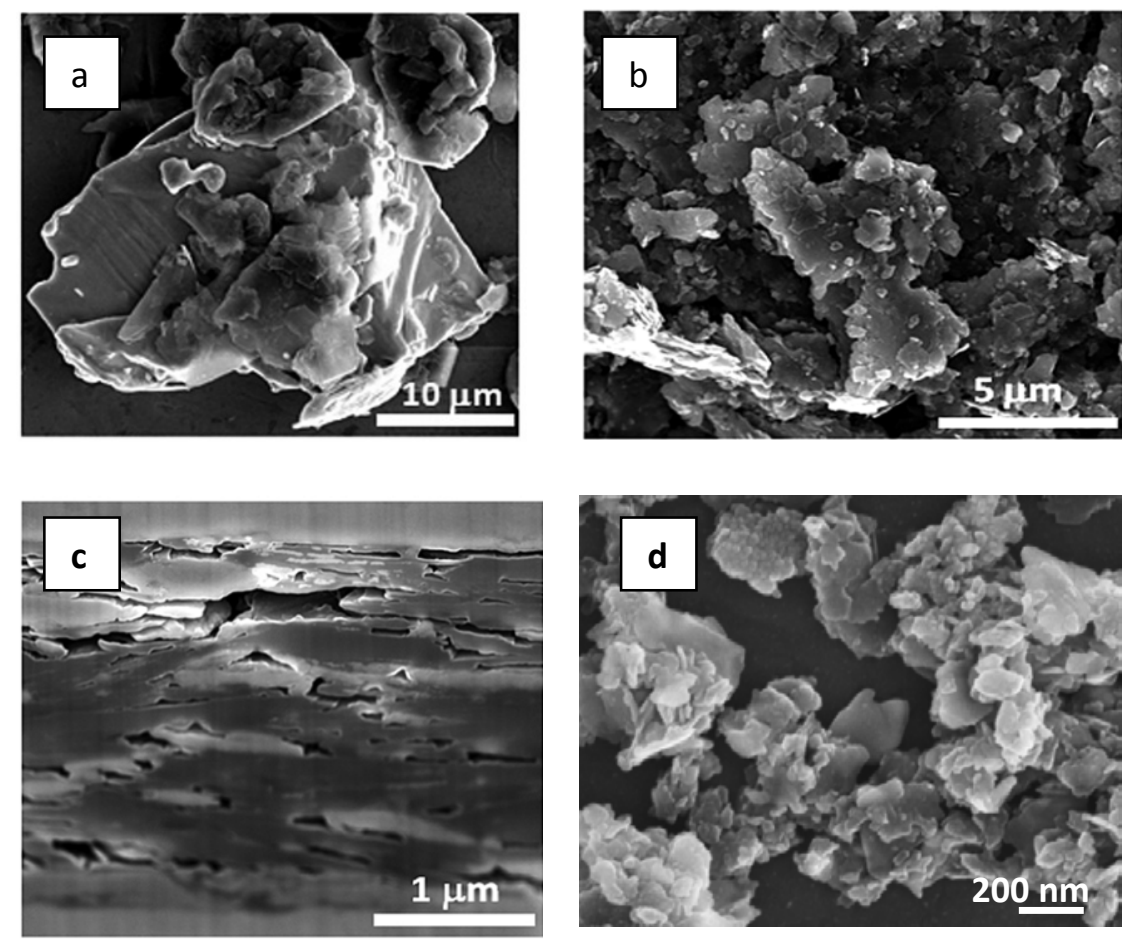

Figure 5 

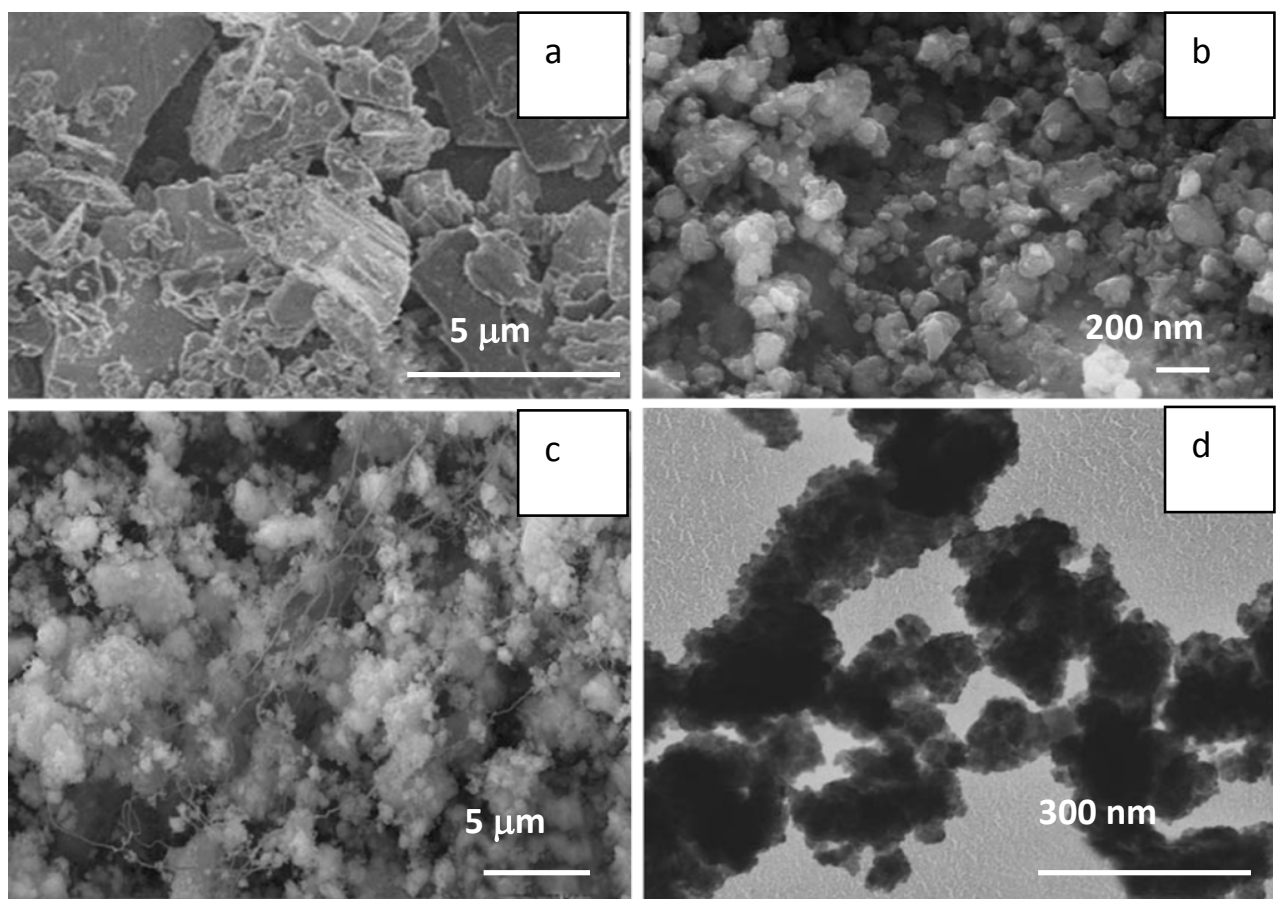

Figure 6 


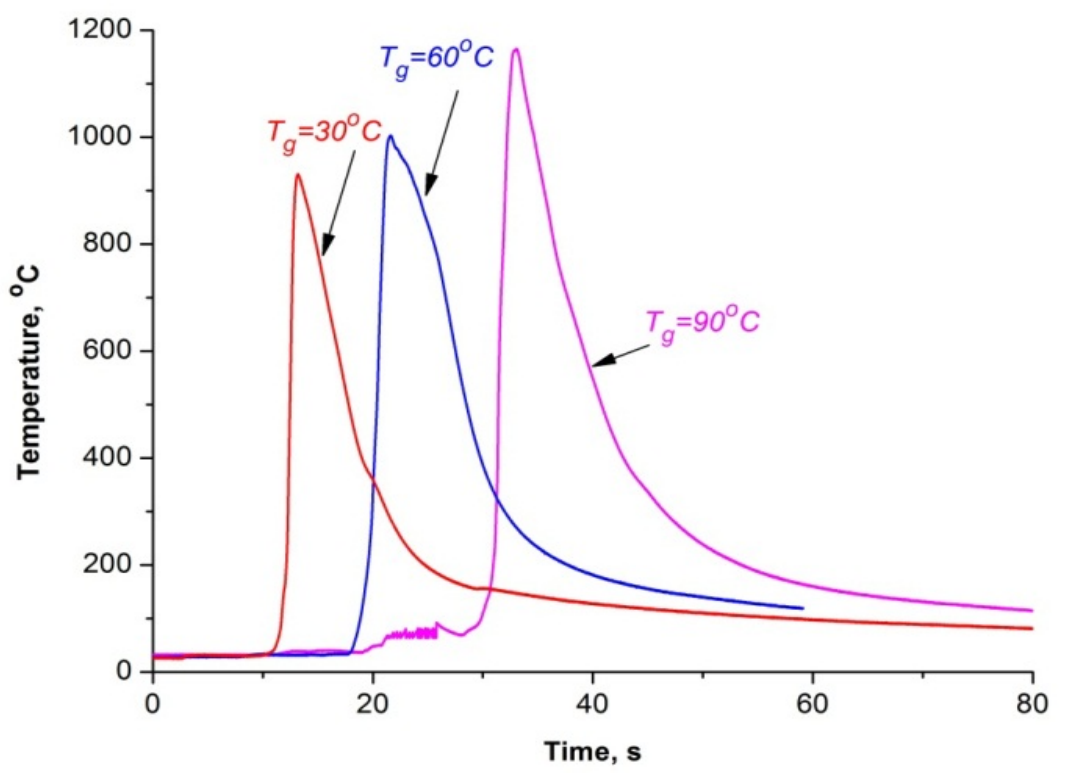

Figure 7 

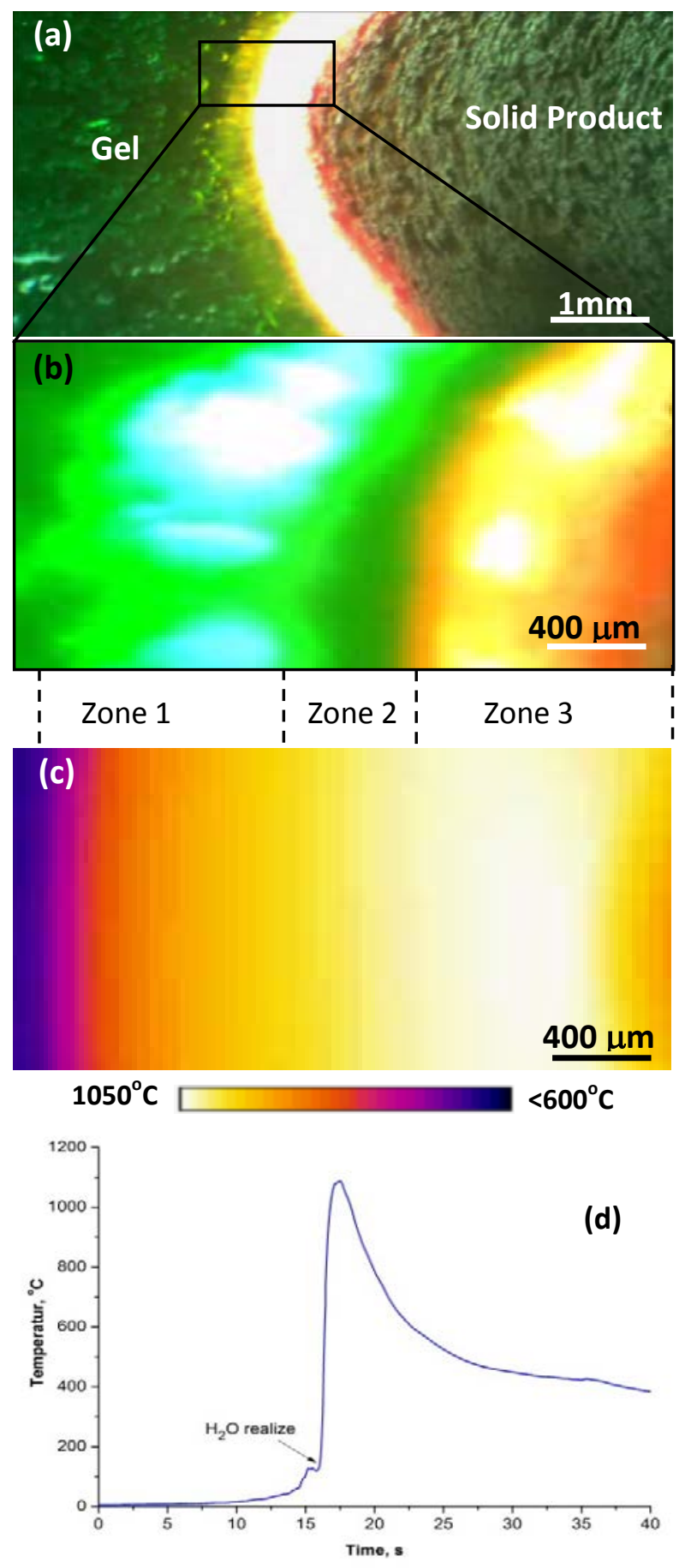

Figure 8. 


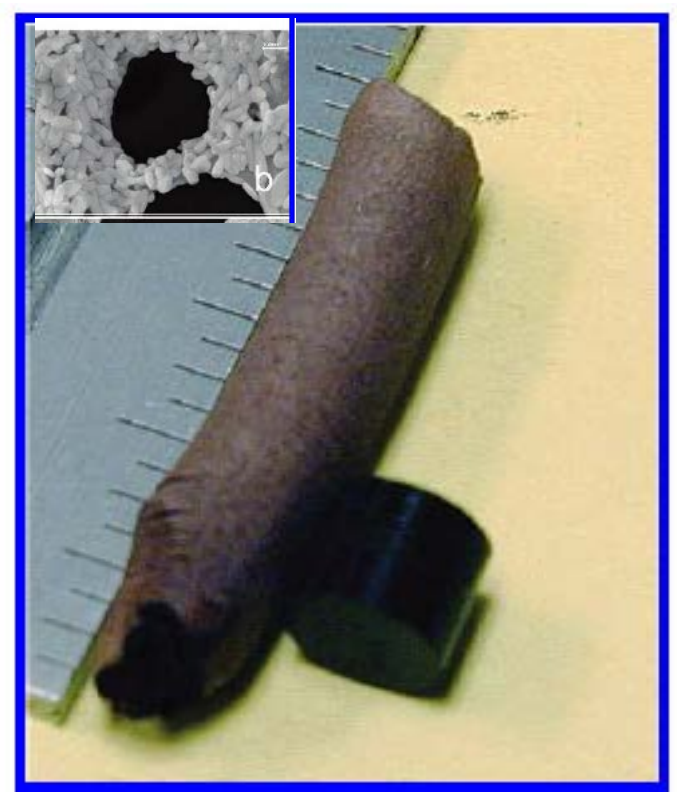

Figure 9 

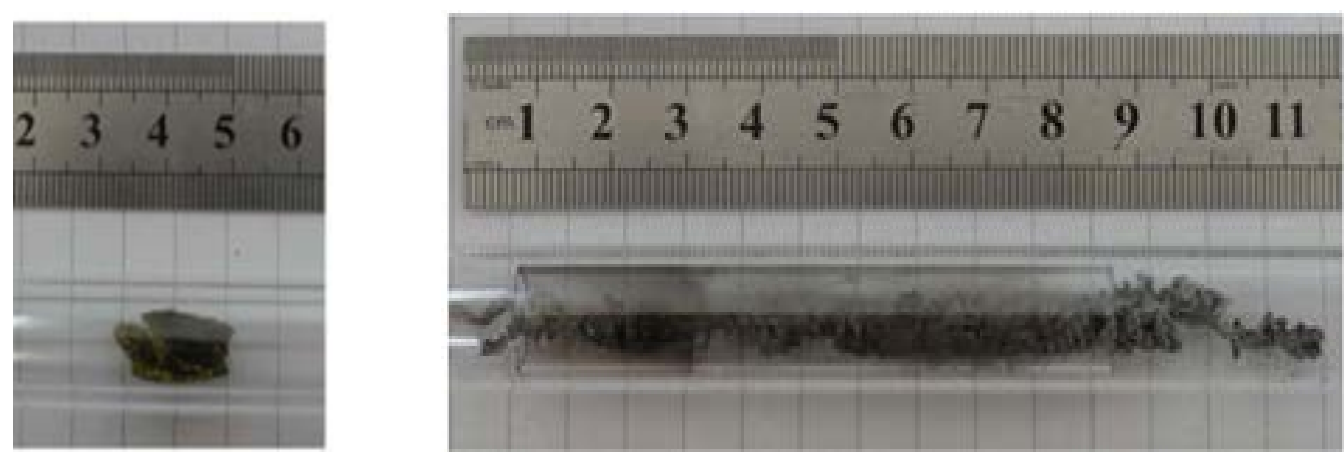

Figure 10 


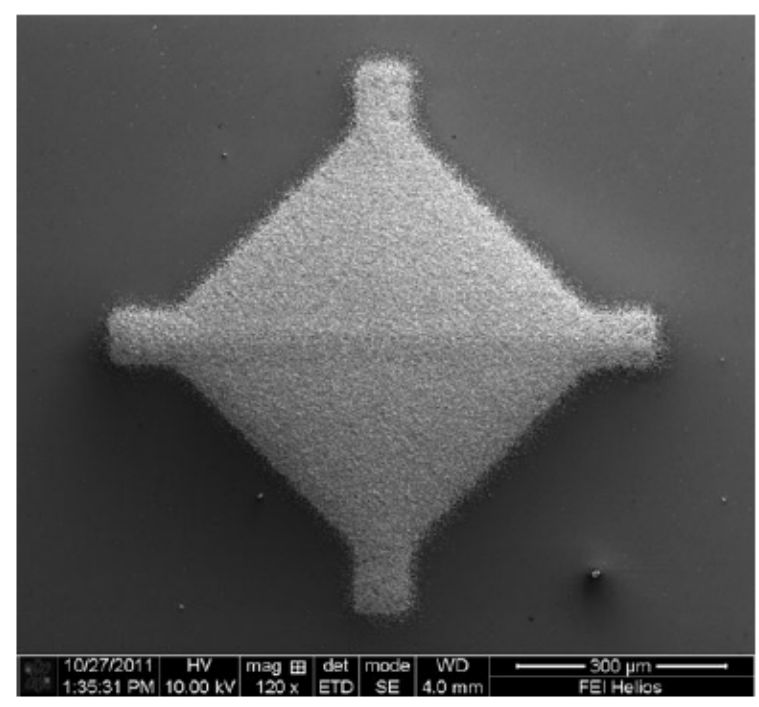

Figure 11 


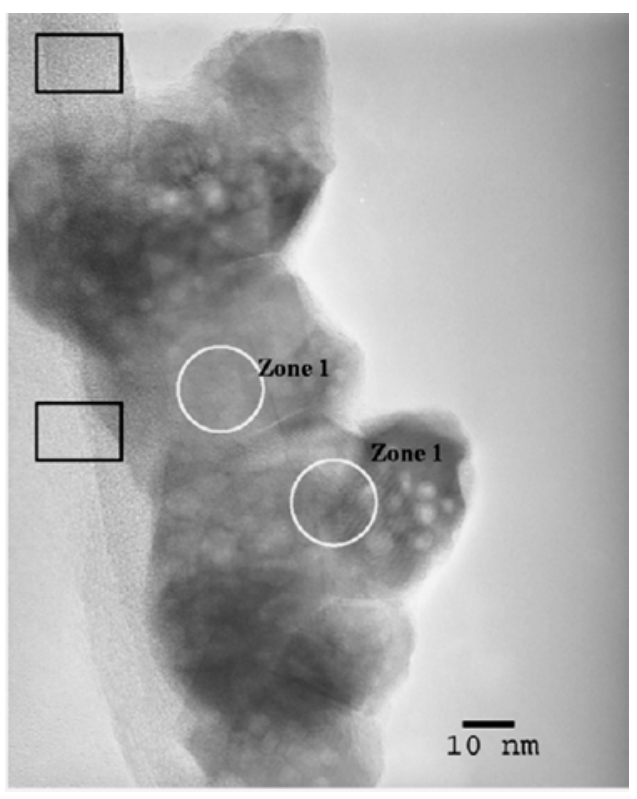

Figure 12 

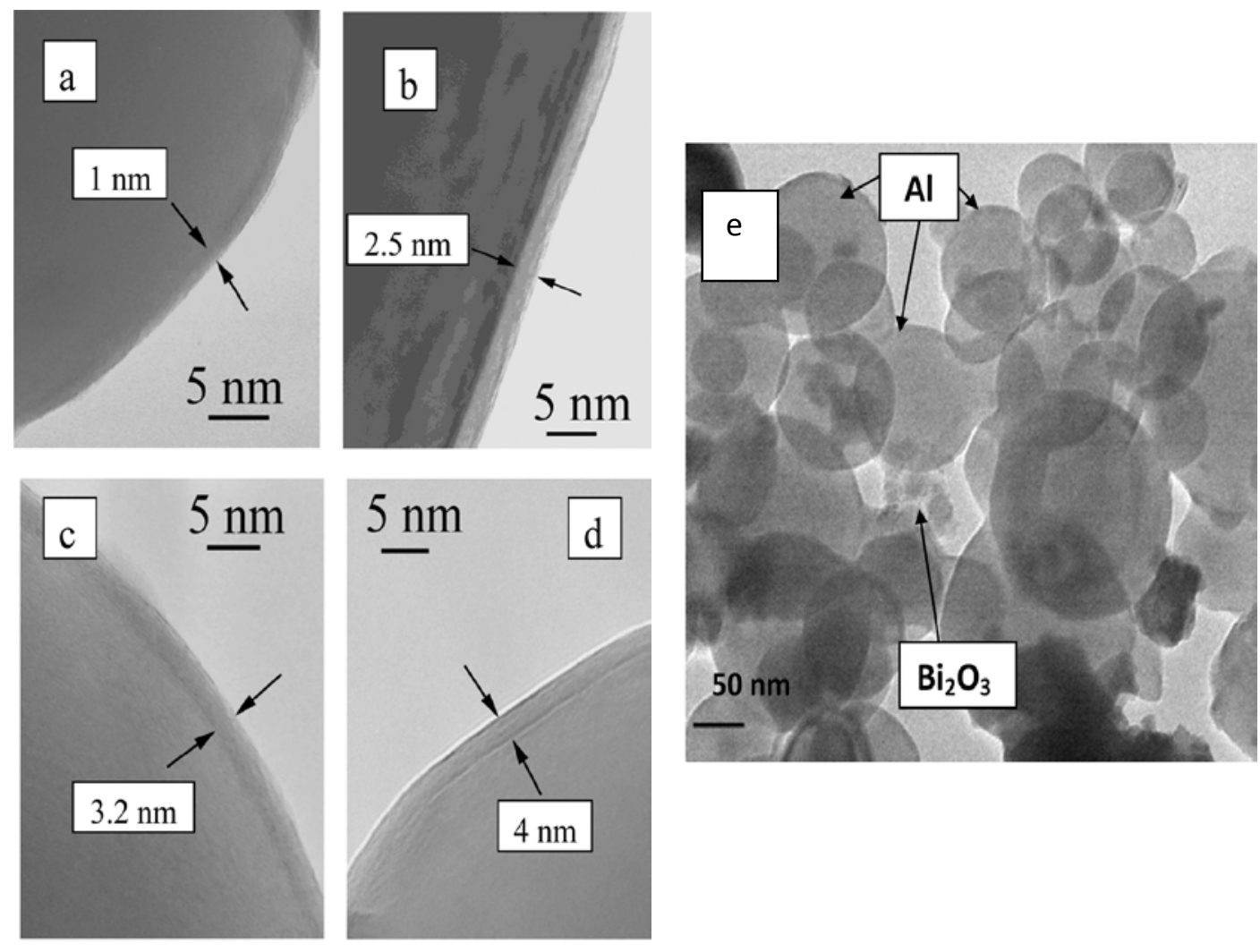

Figure 13 

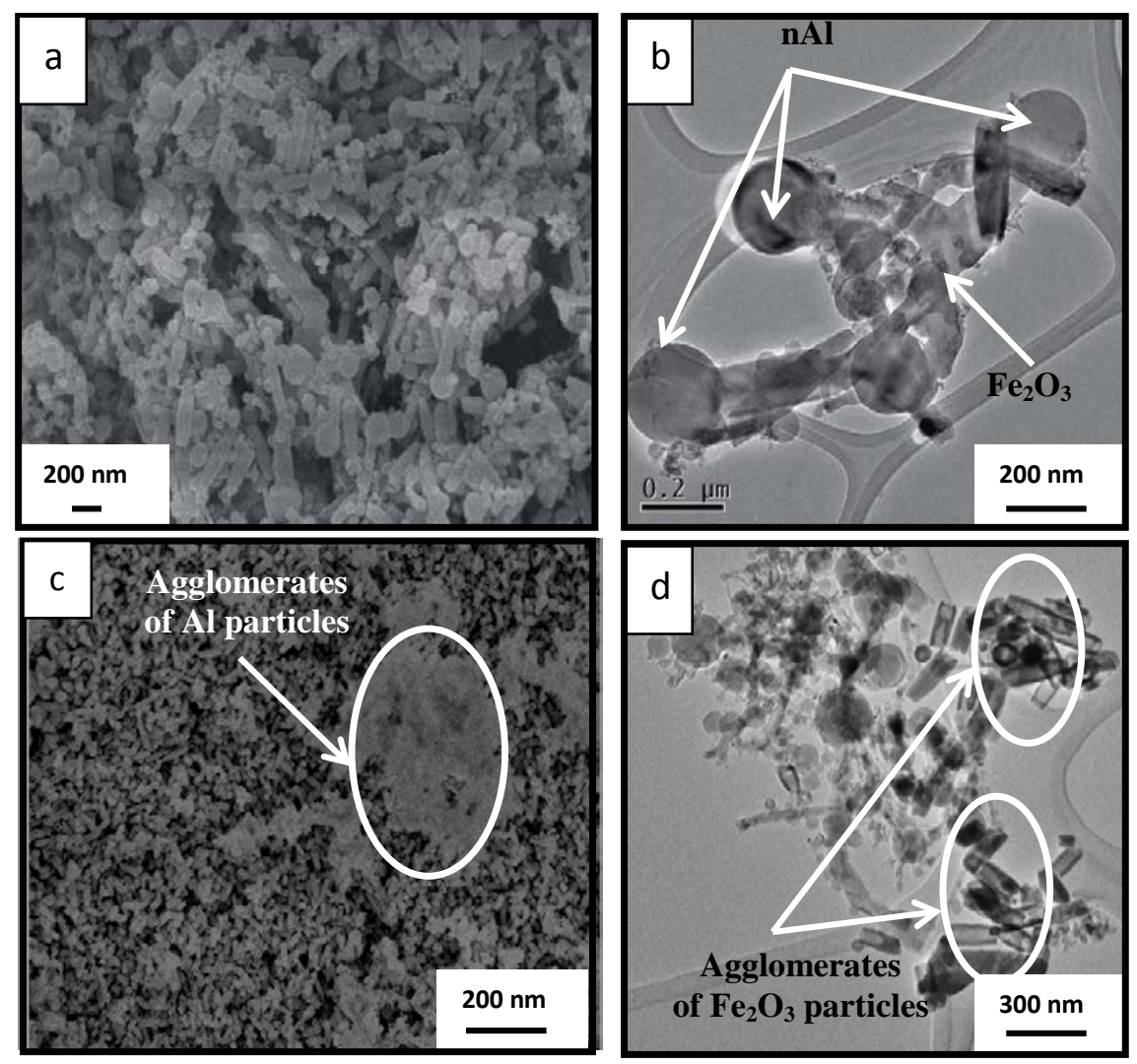

Figure 14 

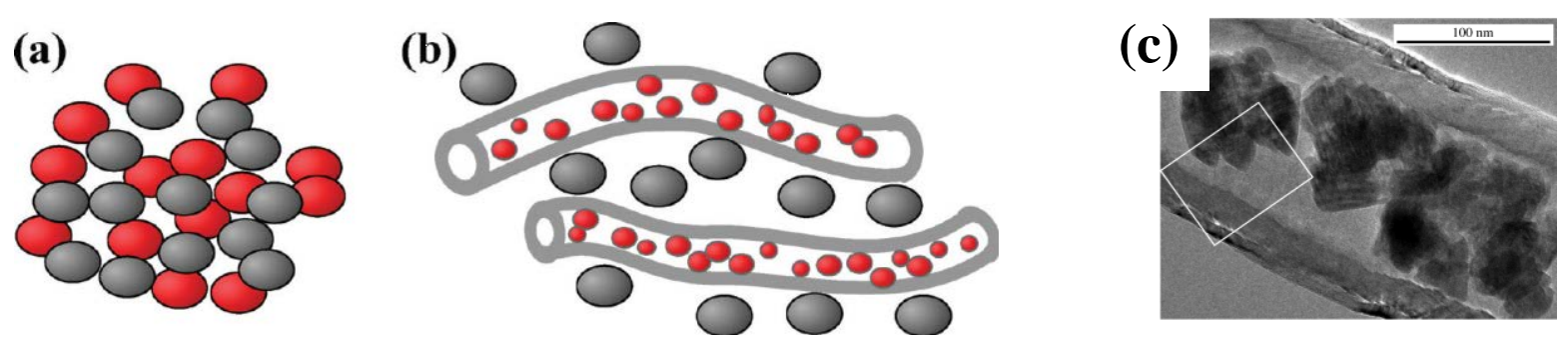

Figure 15 


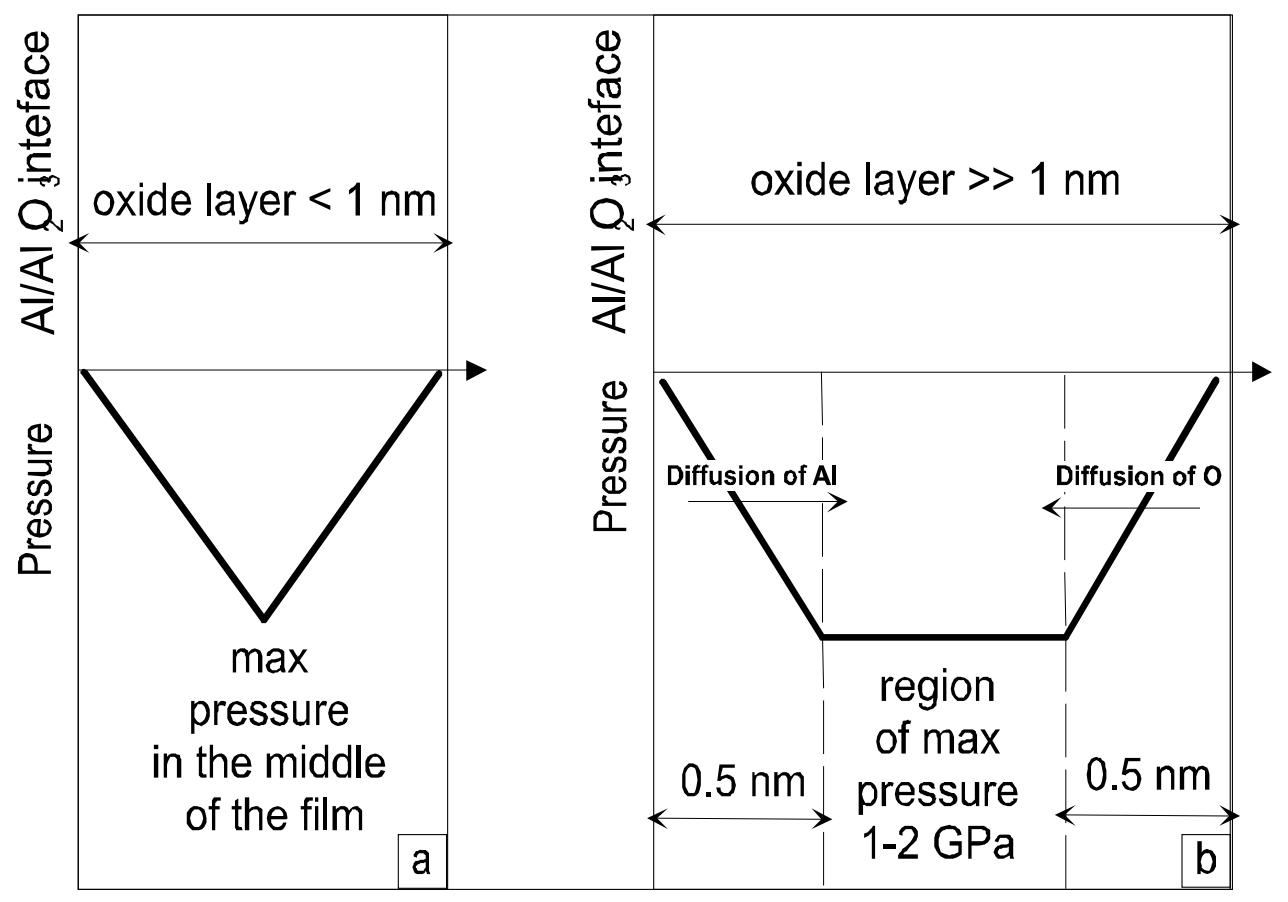

Figure 16 


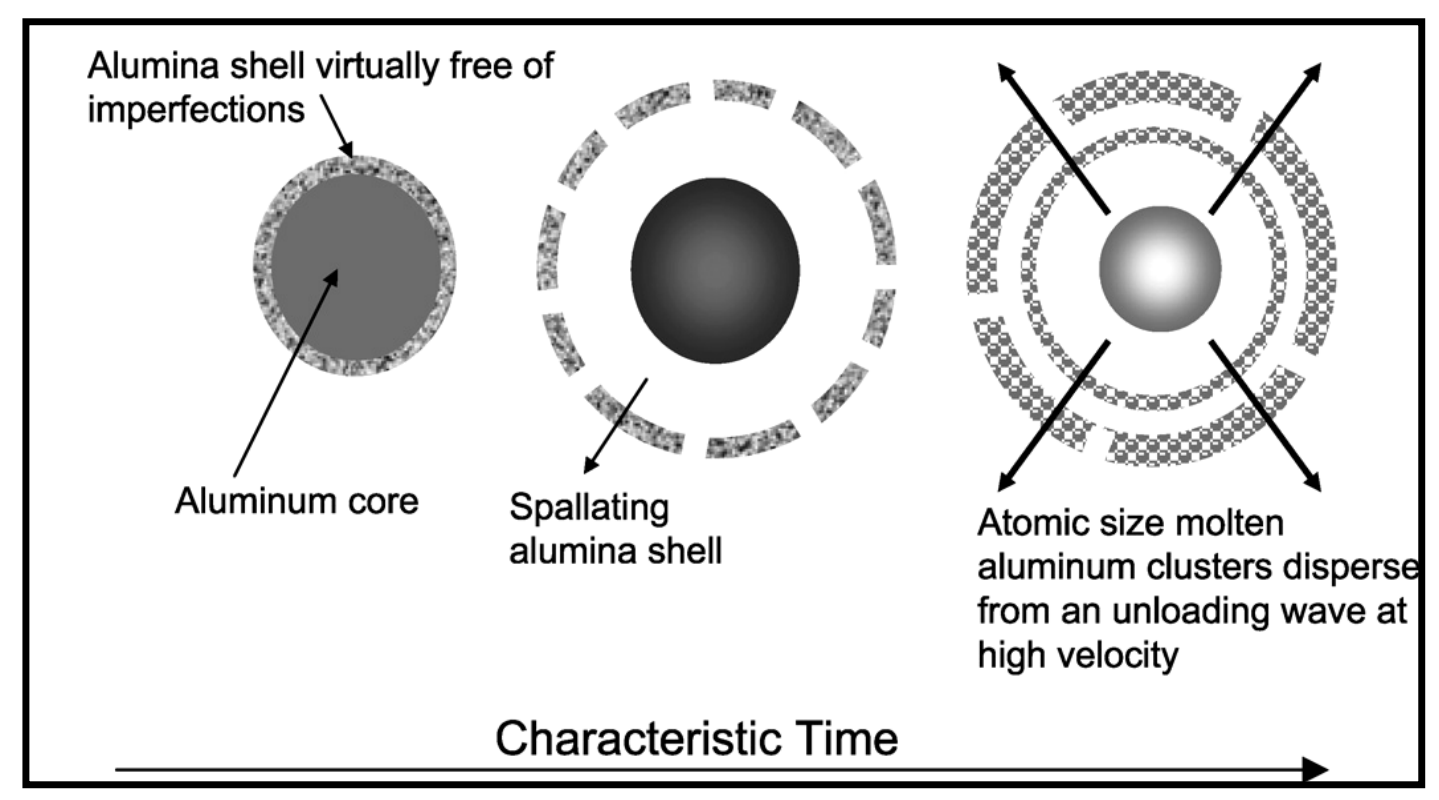

Figure 17 


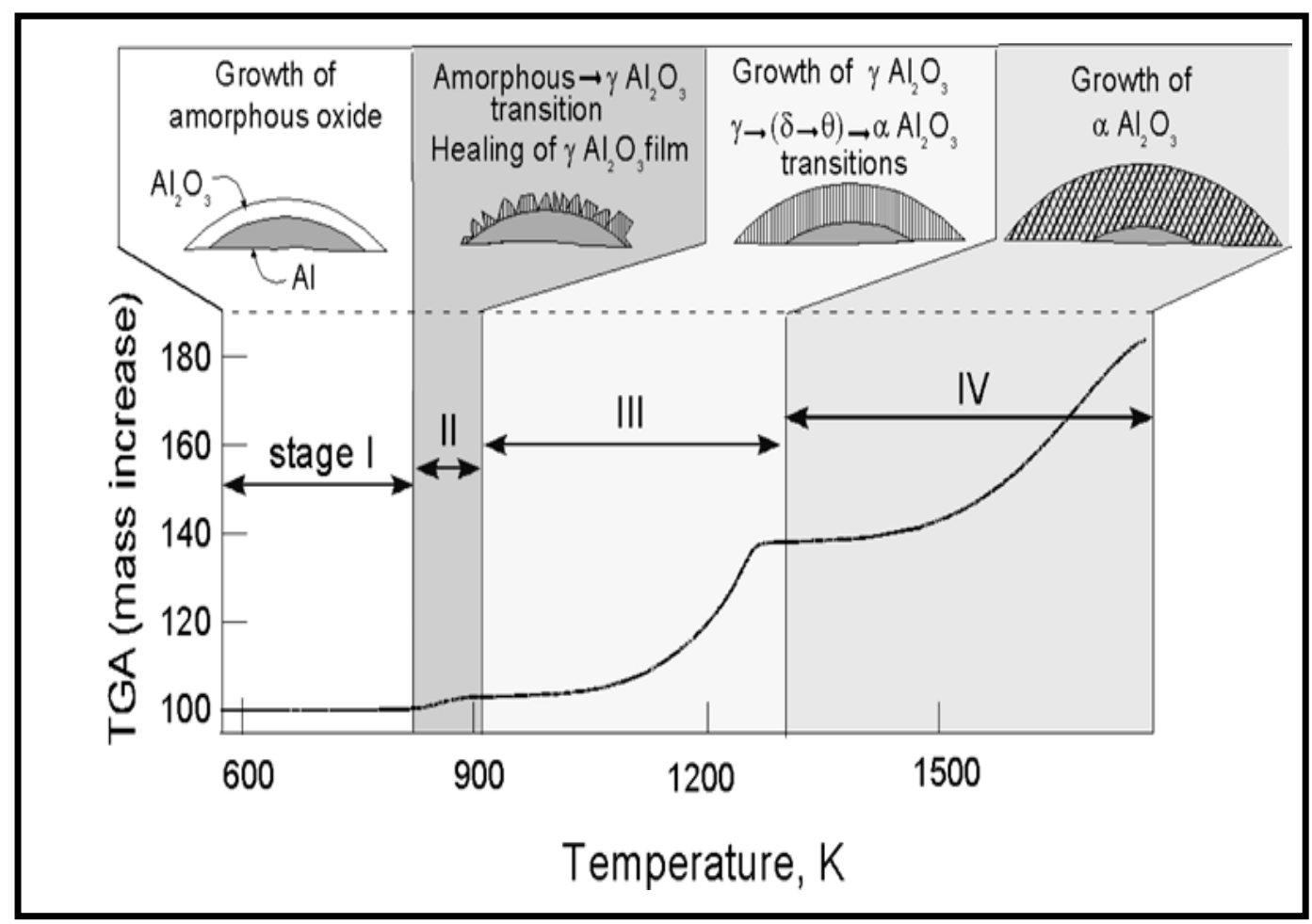

Figure 18 

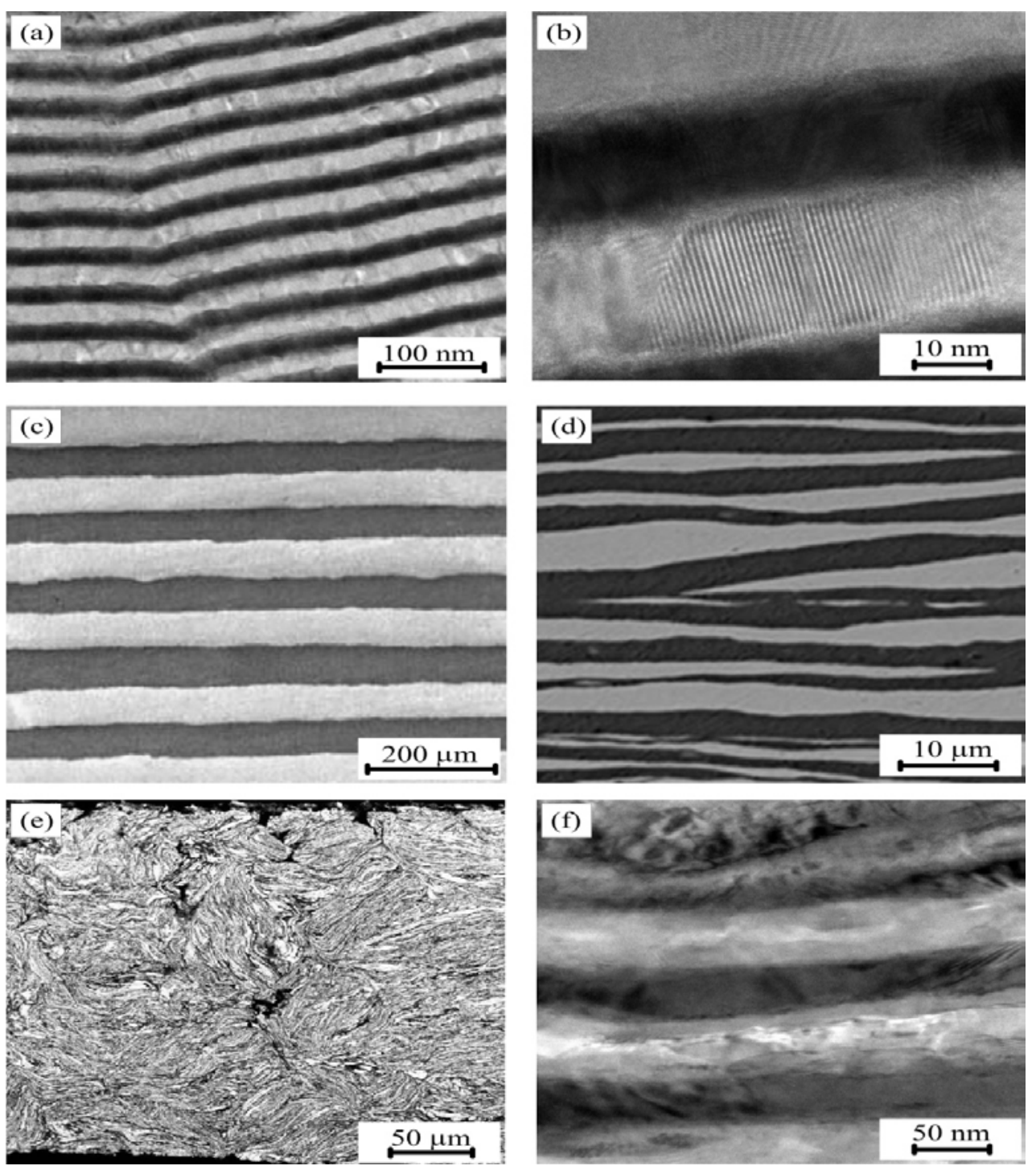

Figure 19 
a

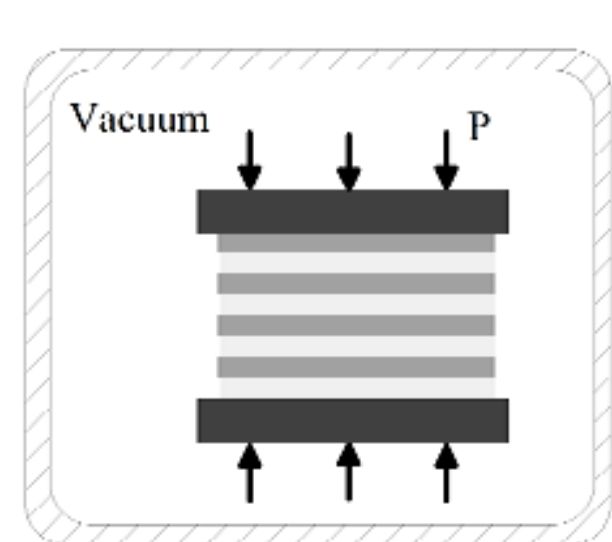

Furnace

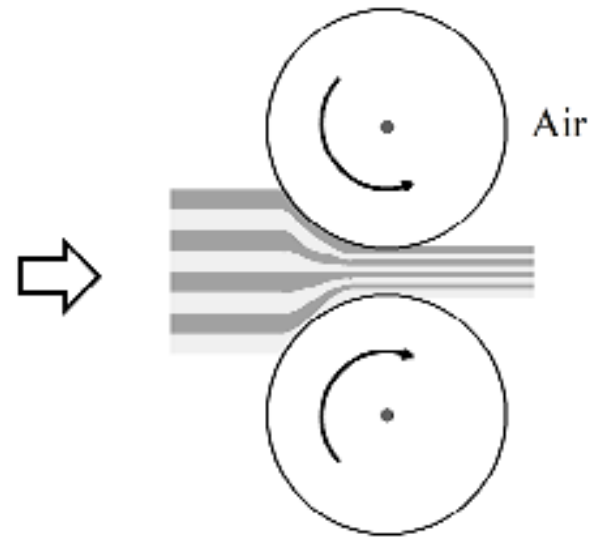

Cold rolling

b

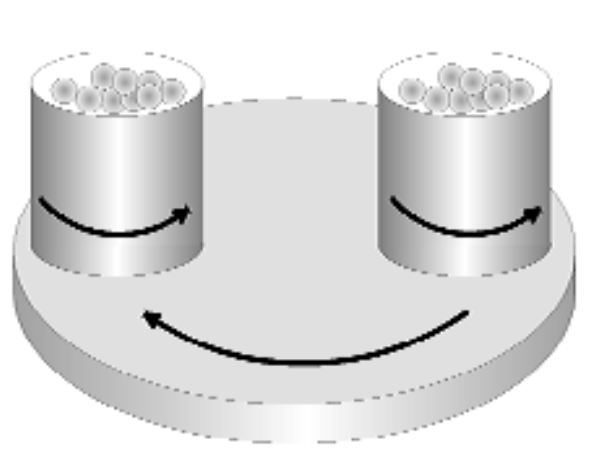

Planetary Ball Mill
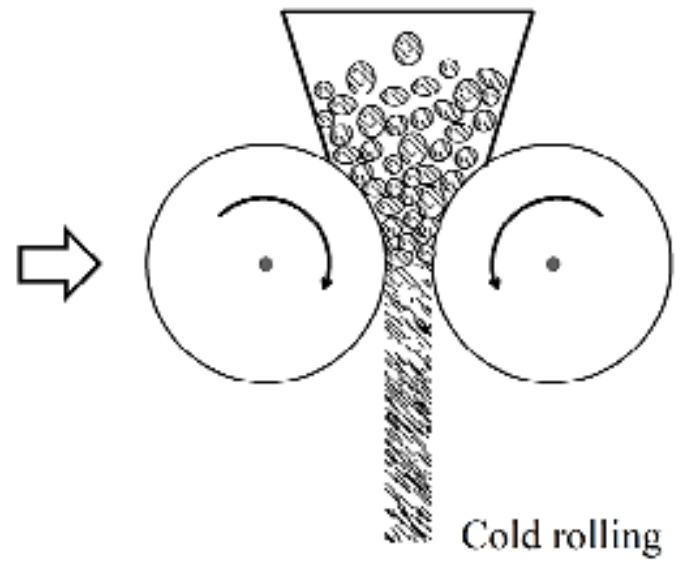

Figure 20 